\title{
RESEARCH
}

Open Access

\section{Small extracellular vesicles secreted by human iPSC-derived MSC enhance angiogenesis through inhibiting STAT3- dependent autophagy in ischemic stroke}

Yuguo Xia ${ }^{1}$, Xiaozheng Ling ${ }^{1}$, Guowen Hu', Qingwei Zhu', Juntao Zhang ${ }^{2}$, Qing Li², Bizeng Zhao ${ }^{2}$, Yang Wang ${ }^{2^{*}}$ and Zhifeng Deng ${ }^{1 *}$ (1)

\begin{abstract}
Background: Small extracellular vesicles (sEV) secreted by mesenchymal stem cells (MSC) derived from human induced pluripotent stem cells (iPSC, iMSC-sEV) are considered to have great potential in treating ischemic diseases. Angiogenesis play an important role in post-stroke recovery. However, no studies have yet been conducted to systemically examine the effect and the underlying mechanism of iMSC-sEV on angiogenesis under brain ischemia conditions.

Methods: Ischemic stroke model was performed in rats induced by middle cerebral artery occlusion (MCAO), and the pro-angiogenic capacity of iMSC-sEV was measured. The in vitro effects of iMSC-sEV on the migration and tube formation of endothelial cells were investigated, respectively. Autophagy and autophagy-related signaling pathway were detected in vivo and in vitro.

Results: We found that iMSC-sEV significantly reduced infarct volume, enhanced angiogenesis, and alleviated longterm neurological deficits in rats after stroke. We also demonstrated that iMSC-sEV increased migration and tube formation of endothelial cells in vitro. A further mechanism study revealed that the pro-angiogenic effect of iMSCsEV was correlated with a reduction in autophagy. Furthermore, iMSC-sEV significantly activated signal transducer and activator of transcription 3 (STAT3), and suppression of STAT3 abolished iMSC-sEV-induced inhibition of autophagy and promotion of angiogenesis in vivo and in vitro.
\end{abstract}

Conclusions: Taken together, our data indicate that iMSC-sEV promote angiogenesis after ischemic stroke, potentially, by inhibiting autophagy, a process that is partially dependent on STAT3 activation.

Keywords: Human induced pluripotent stem cell-derived mesenchymal stem cells, Small extracellular vesicles, Angiogenesis, Autophagy

\footnotetext{
* Correspondence: wangy63cn@126.com; dengzf63@126.com

${ }^{2}$ Institute of Microsurgery and Extremities, Shanghai Jiao Tong University Affiliated Sixth People's Hospital, No. 600 Yishan Road, Shanghai 200233,

China

'Department of Neurosurgery, Shanghai Jiao Tong University Affiliated Sixth People's Hospital, No. 600 Yishan Road, Shanghai 200233, China
}

(c) The Author(s). 2020 Open Access This article is licensed under a Creative Commons Attribution 4.0 International License, which permits use, sharing, adaptation, distribution and reproduction in any medium or format, as long as you give appropriate credit to the original author(s) and the source, provide a link to the Creative Commons licence, and indicate if changes were made. The images or other third party material in this article are included in the article's Creative Commons licence, unless indicated otherwise in a credit line to the material. If material is not included in the article's Creative Commons licence and your intended use is not permitted by statutory regulation or exceeds the permitted use, you will need to obtain permission directly from the copyright holder. To view a copy of this licence, visit http://creativecommons.org/licenses/by/4.0/ The Creative Commons Public Domain Dedication waiver (http://creativecommons.org/publicdomain/zero/1.0/) applies to the data made available in this article, unless otherwise stated in a credit line to the data. 


\section{Background}

Stroke is a leading cause of mortality and disability worldwide [1]. Till now, tissue plasminogen activator (tPA) and endovascular thrombectomy remain the only two effective therapies to treat ischemic stroke [2]. Still, only a small population of patients benefit from the treatments due to the short therapeutic time window or contraindications [3]. Hence, there is an urgent need for effective therapies. Recent experimental and clinical evidences from independent research groups revealed that angiogenesis is correlated with improved neurological recovery after stroke [4-6]. Angiogenesis occurs in the ischemic penumbra within hours after stroke and lasts for weeks. However, ischemic stroke injury stimulated angiogenesis is insufficient to satisfy the need for blood supply and removal of necrotic debris [7]. Hence, developing new and better therapy to augment angiogenesis is of utmost importance for ischemic stroke.

Accumulating studies have reported that cell-based therapy, especially stem cells including embryonic stem cells (ESC), neural stem/precursor cells (NSC/NPC), mesenchymal stem cells (MSC), and induced pluripotent stem cells (iPSC), exerted neuroprotection effect in preclinical stroke research [8]. Among them, MSC are one of the widely studied cells in animal model of ischemic stroke. MSC, with or without modification, have shown remarkable potential of promoting angiogenesis in preclinical ischemic disease studies $[9,10]$ and clinical trial [11], while using tissue-derived MSC sources including bone marrow, adipose, umbilical cord, muscle, dental pulp, and so on [12] as cell-based therapy has its limitations including invasive procurement procedure (bone marrow biopsy or liposuction), limited cell proliferation potential, age-associated functional decline, and potential risk of rejection for allogeneic cell transfer [12, 13], restricting the clinical applications of MSC. With the advent of reprogramming technique, iPSC have been generated successfully from patients' adult somatic cells [14]. Indeed, iPSC have attractive features, for example, iPSC possess unlimited self-renewal and differentiation capacity which could provide a large amount of cells [15], and they can generate unlimited early-passage patient-specific MSC with consistent quality [16]. We and other research groups have recently derived MSC from iPSC, providing a new source of MSC (iMSC) [16, 17]. iMSC have been proven to be alike adult MSC in morphology, surface marker expression profile, global gene expression, tri-lineage differentiation capability, and function [18]. Moreover, iMSC overcome the limitations of tissue-derived MSC, which become a promising alternative for stem cell therapy.

Increasing studies have indicated that the efficacy of MSC therapy against stroke might be attributable to the paracrine activity $[19,20]$ and that small extracellular vesicles (sEV), lipid bilayer nanoparticles containing proteins, lipids, nucleic acid, and other biomolecules, play an important role in this mechanism $[20,21]$. sEV are more stable than stem cells under various physiological conditions [22] and can easily cross the blood-brain barrier $(\mathrm{BBB})$ [21], making them suitable for therapeutic interventions for ischemic stroke. Indeed, Doeppner et al. found that MSC-sEV showed a comparable effect on promoting neurogenesis and angiogenesis after stroke with their parental cells [23]. Our group recently demonstrated that iMSC-sEV could attenuate limb ischemia by promoting angiogenesis [24]. However, to date, there is no report on the application of iMSC-sEV to enhance angiogenesis under ischemic stroke conditions.

Recent evidence has suggested that angiogenic behavior of endothelial cells is in a tight relationship with cell autophagy in vitro and in vivo [25]. Autophagy is a dynamic process of subcellular degradation. Study indicated that autophagy protects against ischemic brain injury by removing the accumulated damaged proteins and organelles which can be recycled for cellular defenses and energy generation [26], while autophagy during ischemic stroke is not always protective. Prolonged and excessive autophagy promotes the progressive consumption of cellular constituents and leads to autophagic cell death [27]. Therefore, suppression of detrimental autophagy may be a target for ischemic brain injury. Indeed, autophagy was observed in brain endothelial cells after stroke insult $[28,29]$. Some studies suggested that cellular autophagy may inhibit the angiogenesis in endothelial cells [30]. However, the relationship between iMSC-sEV and autophagy in the process of angiogenesis after ischemic stroke remains unclear. In the present study, we investigated the pro-angiogenic effect of iMSC-sEV on a rat model of stroke and further explored the potential mechanism. Here, we show for the first time that iMSC-sEV possess the potential to promote angiogenesis after stroke, at least in part, by inhibiting STAT3-dependent autophagy.

\section{Materials and methods}

\section{Generation, culture, and identification of iMSC}

The use of human iPSC in this study was approved by the local ethics committee of the Shanghai Sixth People's Hospital affiliated with Shanghai Jiao Tong University. The generation of mesenchymal stem cells from human induced pluripotent stem cells was previously described [16] with a few modifications. The human iPSC line (iPS-S-01) used in this study was from Institute of Biochemistry and Cell Biology of the Chinese Academy of Sciences in agreement with Liao and Xiao [31]. Six-well plate was pre-coated with vitronectin (Nuwacell ${ }^{\mathrm{TM}}$ VTN, Nuwacel Biotechnology, RP01002) in a concentration of $1 \mu \mathrm{g} / \mathrm{cm}^{2}$ in DMEM/F12 at room temperature for at 
least an hour. Next, iPSC were cultured in vitronectincoated 6-well plate with iPSC culture medium containing basal medium (Nuwacell ${ }^{\mathrm{Tm}}$ Nova Basal Medium, Nuwacell Biotechnology, RP01001-1) and supplement Nuwacell $^{\mathrm{Tw}}$ Nova Supplement, Nuwacell Biotechnology, RP01001-2). When cells reached to 90\% confluency, the culture medium was changed to MSC culture medium containing basal medium (Nuwacell ${ }^{\mathrm{Tm}}$ Nova Missoin Basal Medium, Nuwacell Biotechnology, RP020101) and supplement (Nuwacell $^{\mathrm{Tm}}$ Nova Missoin Supplement, Nuwacell Biotechnology, RP02010-2). Culture medium was changed every 2 days for 14 days. The cells were then trypsinized with $0.25 \%$ trypsin/ $1 \mathrm{mM}$ EDTA (Gibco) and seeded to $25-$ and $75-\mathrm{cm}^{2}$ cell culture flasks (Corning) at a density of $1 \times 10^{5} / \mathrm{mL}$ in MSC culture medium mentioned above. The cells were sub-cultured every 2-3 days when cell reached to $85 \%$ confluency. The morphology of cells was changed to fibroblast-like cells at passage 4 , and the cells were utilized to identify iMSC phenotypical characteristics and tri-lineage differentiation ability [16]. Passages 5 to 10 were used for the following experiments.

\section{Multipotent differentiation potential of iMSC}

Tri-lineage differentiation capability of iMSC was examined as previously described [16]. Briefly, to detect osteogenesis, iMSC culture medium was switched to osteogenesis medium (Gibco) when $90 \%$ confluency was reached. After culture for 21 days, cells were fixed with $4 \%(\mathrm{w} / \mathrm{v})$ paraformaldehyde (PFA) and Alizarin Red staining was used to detect mineralized calcium. To detect adipogenesis, iMSC were cultured under adipogenesis medium (Gibco) for 21 days, followed by Oil Red $\mathrm{O}$ staining. To detect chondrogenesis, $1 \times 10^{6}$ cells were pelleted in a $15-\mathrm{mL}$ polypropylene tube after centrifugation, and chondrogenic medium (Gibco) was gently added to the pellet. After 28 days, the pellet was fixed with $4 \%(\mathrm{w} / \mathrm{v})$ PFA and embedded in optimum cutting temperature compound (OCT) (Thermo Fisher, Waltham, MA, USA). Cryosections $(8 \mu \mathrm{m})$ were cut with freezing microtome (Leica, CM1950, Germany) and stained with Toluidine Blue to examine the presence of proteoglycans. The cells cultured in MSC culture medium were served as control. All images were captured under an optical microscope (Leica, DM6B, Germany).

\section{Flow cytometry analysis}

Flow cytometry was used to identify phenotypical markers of iMSC. Single-cell suspension was collected, and cell number was counted. Cells were then incubated with $1 \%(\mathrm{w} / \mathrm{v})$ bovine serum albumin (BSA) (Gibco) to block the non-specific antigens. Next, $1 \times 10^{6}$ cells were stained with the following conjugated mouse monoclonal antibodies (BD Biosciences): CD73-PE (1: 100, 561014), CD29-PE (1:100, 561795), CD44-FITC (1: 100, 560977), CD146-PE (1:100, 561013), CD34-APC (1: 100, 560940), CD45-FITC (1:100, 560976), CD133-PE (1: 100, 566594), and HLA-DR-PE (1:100, 560943). Nonspecific fluorescence was determined by incubation of similar cell aliquots with isotype-matched mouse monoclonal antibodies (BD Biosciences). After two washes in $1 \%(\mathrm{w} / \mathrm{v}) \mathrm{BSA}$, the cells were resuspended in $300 \mu \mathrm{L}$ of $1 \%$ BSA and analyzed by CytoFLEX flow cytometer (Beckman Coulter Life Science, USA).

\section{Isolation and morphological identification of iMSC-sEV}

$\mathrm{sEV}$ were isolated from the cell culture medium of iMSC by differential ultracentrifugation protocols as previously described [32]. Briefly, the obtained medium was centrifuged at $300 \mathrm{~g}$ for $10 \mathrm{~min}$ and $2000 \mathrm{~g}$ for $10 \mathrm{~min}$ to remove cells, dead cells respectively. After centrifugation at 10, $000 \mathrm{~g}$ for $1 \mathrm{~h}$, the supernatant was filtered through a 0.22 $\mu \mathrm{m}$ filter sterilize Steritop ${ }^{\mathrm{ma}}$ (Millipore) to remove cellular debris and microvesicles (MV). The collected medium was further ultracentrifuged at $100,000 \mathrm{~g}$ for $70 \mathrm{~min}$. After removal of the supernatant, the pellet was resuspended in phosphate buffer saline (PBS), followed by another ultracentrifugation at $100,000 \mathrm{~g}$ for $70 \mathrm{~min}$. Finally, pelleted sEV were resuspended in PBS.

\section{Nano-flow analysis of iMSC-sEV}

The size and concentration of the iMSC-sEV were assessed using nano-flow cytometer (N30 Nanoflow Analyzer, NanoFCM Inc., Xiamen, China) as previously described [33]. Briefly, the side scatter intensity (SSI) was measured by the loading of the standard polystyrene nanoparticles $(200 \mathrm{~nm})$ with a concentration of $1.58 \times$ $10^{8} / \mathrm{mL}$ to the nano-flow cytometer. Next, isolated iMSC-sEV sample diluted with 1000-fold PBS (for a nanoparticle concentration of approximately $5 \times 10^{9} / \mathrm{mL}$ ) was loaded to the nano-flow to measure the SSI. Finally, the concentration of sEV was calculated according to the ratio of SSI to particle concentration in the standard polystyrene nanoparticles. For size measurement, standard silica nanoparticles with mixed size $(68 \mathrm{~nm}, 91 \mathrm{~nm}$, $113 \mathrm{~nm}, 155 \mathrm{~nm}$ ) were load to the nano-flow cytometer to generate a standard cure, followed by the loading of sEV sample. The size distribution was calculated according to the standard cure.

\section{Animal model and iMSC-sEV administration}

All animal experiments were approved by the Animal Research Committee of the Shanghai Sixth People's Hospital (SYXK [Shanghai, China] 2011-0128, 1 January 2011). Male Sprague Dawley (SD) rats (6-8 weeks old, 250-300 g) were randomly assigned to sham or transient middle cerebral artery occlusion (MCAO) groups with 
different treatments (vehicle or iMSC-sEV tail veil injection) by using a lottery drawing box. Transient focal cerebral ischemia was induced by $2 \mathrm{~h}$ MCAO as previously described [34]. Rats in the sham group underwent the same procedure without vascular occlusion. Rectal temperature was monitored and maintained at $37.0 \pm$ $0.5^{\circ} \mathrm{C}$ during the entire procedure using a temperaturecontrolled heating pad. Rats showing no neurological deficits or dead post-MCAO were excluded for data analysis. iMSC-sEV $\left(1 \times 10^{11}\right.$ particles in $500 \mu \mathrm{L}$ PBS $)$ or vehicle (PBS alone, $500 \mu \mathrm{L}$ ) were administered via tail vein injection $4 \mathrm{~h}$ after MCAO based on our previous experience [35].

\section{In vitro culture of human umbilical vein endothelial cells (HUVECs)}

In this study, HUVECs were isolated from human umbilical cords as previously described [16], which were obtained with informed consent and approval by the local ethics committee of the Shanghai Sixth People's Hospital affiliated to Shanghai Jiao Tong University. Briefly, the cords were washed twice with warm PBS to flush out blood and clots; HUVECs were digested with $0.5 \mathrm{mg} / \mathrm{mL}$ type II collagenase (Sigma-Aldrich, St. Louis, MO, USA) for $30 \mathrm{~min}$ at $37^{\circ} \mathrm{C}$ and drawn out from the vessel wall by medium 200 (M200, Gibco) containing 10\% (v/v) fetal bovine serum (FBS, Gibco Life Technologies). After centrifugation at 1000 revolutions per minute (rpm) for 5 min at room temperature, HUVECs were seeded into $1 \%$ $(\mathrm{w} / \mathrm{v})$ gelatin-coated $25-\mathrm{cm}^{2}$ cell culture flasks in M200 with $10 \%$ (v/v) FBS. Once HUVECs reached to $90 \%$ confluency, they were further trypsinized and reseeded into $25-\mathrm{cm}^{2}$ cell culture flasks and maintained in M200 supplemented with $2 \%$ low serum growth supplement (LSGS, Cascade Biologics, Portland, OR, USA). HUVECs at passage 2 were used in the experiments as described below.

\section{Uptake of iMSC-sEV in vivo and in vitro}

To determine the migration of iMSC-sEV into the brain, iMSC-sEV were stained with DiR (Thermo Fisher, USA) according to the protocol as previously described [36] with small modification. Briefly, sEV were incubated with DiR fluorescent dye under room temperature for $15 \mathrm{~min}$, followed by ultracentrifugation at $100,000 \mathrm{~g}$ in PBS to get rid of the unlabeled dye. Four hours after MCAO procedure, the rats were intravenously administered with a single dose of DiRlabeled sEV $\left(1 \times 10^{11}\right.$ particles in $500 \mu \mathrm{L}$ PBS $)$. Six hours later, the rats were anesthetized and the DiR fluorescent signals were detected using the IVIS Spectrum imaging system (PerkinElmer, USA). In another experiment, freshly isolated iMSC-sEV were labeled with Dio fluorescent dye (Beyotime biotechnology, China, C1038) according to the manufacturer's instructions. Briefly, iMSC-sEV were incubated with $10 \mu \mathrm{M}$ Dio for $30 \mathrm{~min}$ under room temperature and washed with PBS twice. Dio-labeled $\operatorname{sEV}\left(1 \times 10^{11}\right.$ particles in $500 \mu \mathrm{L}$ PBS $)$ were administered intravenously $24 \mathrm{~h}$ after $\mathrm{MCAO}$ and rats were sacrificed $24 \mathrm{~h}$ after injection. Brian cryosections $(20 \mu \mathrm{m})$ were stained with DAPI solution and observed with fluorescence microscope (Leica, DM6B, Germany). In the in vitro experiment, iMSC-sEV were labeled with Dil fluorochrome (Thermo Fisher, USA) according to the manufacturer's protocol with the same incubation and wash procedures mentioned above. Next, Dil-labeled sEV were added into culture medium and incubated with HUVECs for $4 \mathrm{~h}$. In the control group, the same volume and concentration of Dil dye without iMSC-sEV labeling was ultracentrifuged and washed as mentioned above, followed by incubation with HUVECs. Next, culture medium was discarded, and the cells were rinsed twice with PBS prior image capture under the fluorescence microscope (Leica, DM6B, Germany).

\section{EdU administration}

EdU was utilized to trace cell proliferation after MCAO. Briefly, three doses of $50 \mathrm{mg} / \mathrm{kg}$ EdU (Life technologies, USA, e10187) in PBS was intraperitoneally (i.p.) injected in rats at days 3, 5, and 7 after MCAO respectively. Four hours after the last administration of EdU, the rats were sacrificed for later experiments.

\section{Immunofluorescence staining and quantification}

At different timepoint after MCAO as mentioned in specific experiments, the rats were anesthetized and perfused with ice-cold saline, followed by $4 \% \quad(\mathrm{w} / \mathrm{v})$ paraformaldehyde perfusion. Next, brains were removed, fixed in $4 \%$ formaldehyde overnight at $4{ }^{\circ} \mathrm{C}$, and dehydrated with gradient sucrose solutions $(20 \%, 30 \%$, and $35 \%(\mathrm{w} / \mathrm{v}))$. After being embedded and frozen in an optimal cutting temperature compound (OCT), the brains were sliced into $25-\mu \mathrm{m}$-thick coronal sections. The brain sections were then stained with specific markers including CD31 (1:100, Abcam, UK, ab28364), MAP-2 (1:200, Cell Signaling Technology, USA, 4542S), and CD34 (1: 100, Abcam, UK, ab81289). Fluorescence images were acquired using a fluorescence microscope (Leica, DM6B, Germany). For the quantification of $\mathrm{CD} 34^{+}, \mathrm{CD}^{+} 1^{+}$ $\mathrm{EdU}^{+}$cell number, and $\mathrm{CD}^{+} 1^{+}$vascular density, four sections from the region of interest (ROI) were calculated for each rat by ImageJ software (National Institutes of Health, Bethesda, MD, USA). The value from MCAO groups was normalized by the sham group and compared. 


\section{Measurement of brain infarct}

Infarct volume was assessed using a 3-Tesla magnetic resonance imaging (MRI) scanner (Siemens, Germany) at day 2 and day 28 and immunofluorescence staining of microtubule-associated protein 2 (MAP-2) at day 28 post-stroke. Images were analyzed using ImageJ software (National Institutes of Health, Bethesda, MD, USA) by an investigator who was blinded to experimental group assignment. Infarct volume was calculated by adding up the consecutive 8 infarct areas (contralateral area minus the non-hyperintense area of the ipsilateral side) with a 1.5-mm interval in the T2-weighted MRI image. The extent of brain infarct was presented as the percentage of the infarct volume versus the corresponding contralateral brain tissue volume.

\section{Behavior tests}

Behavioral tests were conducted for consecutive 3 days before stroke as a training session and 1, 3, 7, 14, 21, and 28 days after stroke using modified neurological severity score (mNSS) [37] and the foot-fault test [34] as described previously. In brief, mNSS was graded as 0 to 18 , with a higher score representing more severe neurological dysfunction. Foot-fault was used to test the sensorimotor function. Rats were placed on the elevated grid surface. A foot fault was defined as a step slipping off the grid wire. Each animal was recorded for $3 \mathrm{~min}$ per trial per test day. The investigator performing the tests was blinded to group assignment. The data were presented as percentage of foot fault by the contralateral limbs versus total steps.

\section{Morphology identification of sEV and evaluation of autophagy using TEM}

For the observation of morphology in sEV, freshly isolated SEV were loaded onto a continuous carbon grid, fixed in $3 \%(\mathrm{w} / \mathrm{v})$ glutaraldehyde, and stained with $2 \%$ $(\mathrm{w} / \mathrm{v})$ uranyl acetate. For autophagy detection, brain tissues or cell pellets were fixed using $3 \%(\mathrm{w} / \mathrm{v})$ glutaraldehyde for at least $48 \mathrm{~h}$ at $4{ }^{\circ} \mathrm{C}$ and post-fixed with osmium tetroxide. Samples were dehydrated in a graded series of alcohol concentrations, embedded in epoxy resins, and sectioned. Samples were observed using transmission electron microscopy (TEM; H7650, Hitachi, Tokyo, Japan).

\section{Oxygen and glucose deprivation (OGD)}

HUVECs were seeded onto 6-well plate, and OGD was conducted when the confluency reach to $90 \%$. Briefly, the culture medium of the HUVECs was replaced with glucose-free DMEM (Gibco, NY, USA) containing the same supplements as mentioned above without FBS. The HUVECs were then transferred to anaerobic conditions (5\% $\mathrm{CO}_{2}$ and $95 \% \mathrm{~N}_{2}$ ) and incubated for $8 \mathrm{~h}$. OGD was then ended by changing to normal culture medium with FBS and incubated under normoxia conditions (5\% $\mathrm{CO}_{2}$ and $95 \%$ air) with the presence of iMSC-sEV $(1 \times$ $10^{9}$ particles $/ \mathrm{mL}$ ) or vehicle (PBS) for $24 \mathrm{~h}$. Control HUVECs were cultured under normal conditions (5\% $\mathrm{CO}_{2}$ and $95 \%$ air) for the same duration without any treatment. Next, cells were harvested for further analysis.

\section{Transwell assay}

HUVECs were resuspended in culture medium and seeded in the upper transwell chamber with $8 \mathrm{~mm}$ pore size (Corning, Lowell, MA, USA), and HUVECconditioned medium was added to the lower chamber. After incubation for $24 \mathrm{~h}$, the non-migrated cells on the upper surface of the filter were removed with cotton swab. The migrated cells were fixed with methanol and glacial acetic acid (mixed at 3:1) and then stained using crystal violet staining solution. The migrated cells were imaged using an optical microscope, and the average number of cells was counted in six random fields.

\section{Tube formation assay}

The in vitro tube formation was assayed according to the manufacturer's instructions. The $200 \mu \mathrm{l}$ of Matrigel (BD Biosciences, Bedford, MA, USA) solution was added into 48-well plate and incubated at $37^{\circ} \mathrm{C}$ for $30 \mathrm{~min}$. HUVECs in a concentration of $1.5 \times 10^{5}$ per $\mathrm{mL}$ were seeded on the Matrigel and cultured for $24 \mathrm{~h}$. The total tube length was measured by the Image J software.

\section{STAT3 inhibitor treatment}

To inhibit phosphorylation of STAT3 pharmacologically, the static was applied. For the in vivo experiment, stattic with concentration of $3.75 \mathrm{mg}$ per $\mathrm{kg}$ was intravenously injected in rats $4 \mathrm{~h}$ after MCAO operation accompany with iMSC-sEV. For the in vitro part, HUVECs were challenged by OGD for $8 \mathrm{~h}$ and then $5 \mu \mathrm{M}$ of stattic with iMSC-sEV were added to the culture medium to block the activation of STAT3.

\section{Western blot analysis}

For identification of sEV using western blot analysis, three positive markers of ESC-sEV including CD9, TSG101, and Alix, as well as one negative marker GM130, were evaluated. Specifically, iMSC-sEV were collected as described above. iMSC-sEV proteins were harvested using RIPA lysis buffer (Beyotime biotechnology, China, P0013C) supplemented with protease inhibitor cocktail (Beyotime biotechnology, China, ST505). Next, the protein concentration of iMSC-sEV was measured by the Pierce BCA Protein Assay Kit (Beyotime biotechnology, China, P0012). Proteins were then separated by sodium dodecyl sulfate-polyacrylamide gel 
electrophoresis (SDS-PAGE, EpiZyme, China) and transferred to polyvinylidene fluoride membranes (PVDF, Millipore, USA). The membranes were blocked with 5\% (w/v) non-fat milk for $2 \mathrm{~h}$ and incubated overnight under $4{ }^{\circ} \mathrm{C}$ with the following antibodies: rabbit monoclonal anti-CD9 (1:1000, Cell Signaling Technology, USA, 13174s), mouse monoclonal anti-TSG-101 (1: 1000, Abcam, UK, ab83), anti-Alix (1:1000, Cell Signaling Technology, USA, 2171 s), and mouse polyclonal anti-GM130 (1:500, Abcam, UK, ab169276). After three washes with TBST, the membranes were incubated with HRP-conjugated secondary antibodies (1:2000, Cell Signaling Technology, USA) under room temperature for 1 h. The immunoreactive bands were visualized using ECL (Thermo Fisher Scientific, USA, WP20005) and imaged with a FluorChem M Fluorescent Imaging System (ProteinSimple, Santa Clara, CA, USA).

For western analysis of HUVECs in the in vitro experiment, cells were seeded on 6-well plate followed by 8 -h OGD and 24-h reoxygenation (RO) with or without treatment. Control cells were cultured with the same amount of time as other groups, but without any treatment. After that, cells were washed three times with ice cold PBS, followed by adding of RIPA solution to harvest proteins. Protein concentrations were measured using BCA measurement kit. Total amount of $10-\mu \mathrm{g}$ protein of cell lysates were run on SDS-PAGE gels. For the in vivo experiment, rats were perfused with ice-cold PBS and the ipsilateral infarct brain tissue was harvest quickly on ice to avoid protein denature, followed by homogenization and lysis in RIPA solution. Total amount of 30- $\mu \mathrm{g}$ protein of tissue lysates were run on SDS-PAGE gels. Next, proteins were transferred to PVDF membrane and then blocked with 5\% non-fat milk for $2 \mathrm{~h}$, followed by incubation with primary antibodies against $\beta$-actin (1:1000; Abcam, UK, ab133626), LC3 (1:1000; Cell Signaling Technology, USA, 12741), Beclin1 (1:1000; Cell Signaling Technology, USA, 3495), P62 (1:1000; Cell Signaling Technology, USA, 5114), STAT3 (1:1000; Cell Signaling Technology, USA, 9139), and p-STAT3 (1:1000; Cell Signaling Technology, USA, 9145) overnight at $4{ }^{\circ} \mathrm{C}$. After rinse with TBST, membranes were incubated with HRP-conjugated secondary antibodies (1:2000, Cell Signaling Technology, USA) at room temperature for $1 \mathrm{~h}$. Protein level was detected using the ECL detection system. The intensity of each band was analyzed using ImageJ software.

\section{Statistical analysis}

Detailed biological replicates $(\mathrm{N})$ used in each experiment were stated in the figure legend. For western blot (Figs. 4a, d, 5a, c, and 6a, c), tube formation assay (Figs. $3 \mathrm{~g}$ and $7 \mathrm{e}$ and Fig. S5C), and transwell assay (Figs. 3e and $7 \mathrm{c}$ and Fig. S5A), each experiment was repeated for at least 3 times using different batch of cells. Data were presented as mean $\pm \mathrm{SD}$. The Student $t$ test was used to assess the difference between two groups, and the oneway analysis of variance (ANOVA) with the Bonferroni post hoc test was applied for comparisons among multiple groups. Statistical analysis was performed using GraphPad Prism software (version 8.0). Significant difference was considered to be $P$ value $<0.05$.

\section{Results}

\section{Characterization of iMSC and iMSC-sEV}

To identify iMSC, firstly, flow cytometry was applied to evaluate the surface antigen profile of the cells. The results showed that iMSC highly express antigen markers including CD73, CD29, CD44, and CD146, but not CD34, CD45, CD133, and HLA-DR (Fig. S1A), which were typical for MSC. Next, the tri-lineage differentiation ability of iMSC was examined. Highly positive cells were visualized in the Alizarin Red (Fig. S1B), Oil Red O (Fig. S1C), and Toluidine Blue staining (Fig. S1D), which indicates the osteogenic, adipogenic, and chondrogenic abilities of iMSC respectively. All these results revealed that the obtained iMSC showed typical characteristics and held multipotent differentiation capability.

sEV were isolated from the cell culture supernatant of iMSC and identified using TEM, nano-flow cytometer, and western blot analysis. TEM analysis showed that iMSC-sEV were typical cup-shaped vesicles (Fig. 1a). Nano-flow analysis revealed that the average diameter was ranging from 60 to $160 \mathrm{~nm}$, and the concentration of the iMSC-sEV was approximately $1.36 \times 10^{11}$ particles/mL (Fig. 1b). Western blot analysis determined the presence of exosomal markers, such as CD9, TSG101, and Alix, whereas the cis-Golgi matrix protein GM130 was not detected (Fig. 1c). These data suggested that we had successfully isolated iMSC-sEV.

\section{iMSC-sEV reduce ischemic brain injury and improve neurological function after MCAO}

Firstly, we determined whether intravenous administration of iMSC-sEV could migrate into the brain. iMSC$\mathrm{sEV}$ were labeled with DiR fluorescent dye and administered to rats $4 \mathrm{~h}$ after MCAO. While Most of the DiR signals were showed in the lung, liver, spleen, and kidney (data no shown); DiR-labeled iMSC-sEV-treated rats exhibited visible fluorescence in the brain compared to vehicle-treated rats, indicating the ability of iMSC-sEV to cross the blood-brain barrier (BBB) and migrate into the brain (Fig. S2A). Besides, in another set of experiment, Dio-labeled iMSC-sEV were used to track the distribution in the brain. From Fig. S2C, positive fluorescence signals were observed in cells at the periinfarct area as well as the corresponding contralateral hemisphere, further confirming the previous finding that 


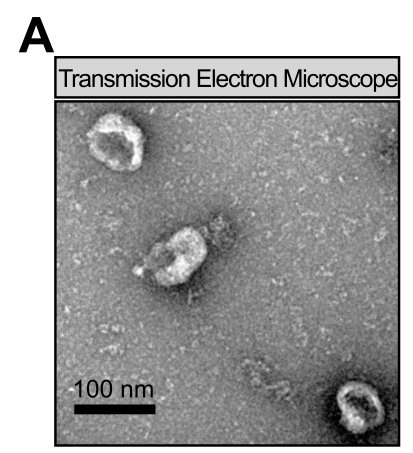

B
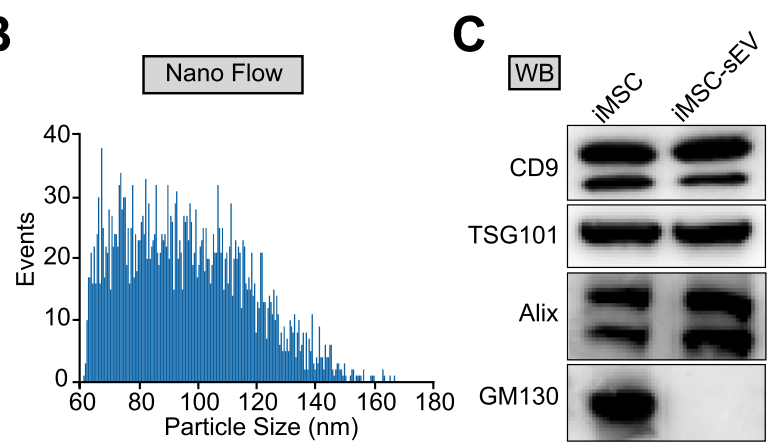

Fig. 1 Characterization of iMSC-sEV. a Representative image of iMSC-sEV observed by TEM. Scale bar $=100 \mathrm{~nm}$. b The particle size distribution of iMSC-sEV measured by nano-flow cytometer. c Western blotting showing the expression of exosomal markers including CD9, TSG101, and Alix in iMSC-sEV, but not the negative marker GM130

iMSC-sEV could infiltrate into the brain even with the uncompromised BBB.

We then investigated the therapeutic effects of iMSC$\mathrm{sEV}$ in rats after ischemic stroke. Based on our previous experience in treating rat model of ischemic stroke using urinal stem cell derived sEV (USC-sEV) [35], the dose of $1 \times 10^{11} \mathrm{iMSC}$-sEV particles number was chosen for the following in vivo experiment. Brain infarct size was monitored using MRI on day 2 and day 28 and MAP-2 staining on day 28 after MCAO (Fig. 2a, b). No significant difference was visualized for infarct volume between two groups on day 2 after stroke (Fig. 2a, b). However, iMSC-sEV treatment significantly attenuated brain infarct as compared to the vehicle group 28 days

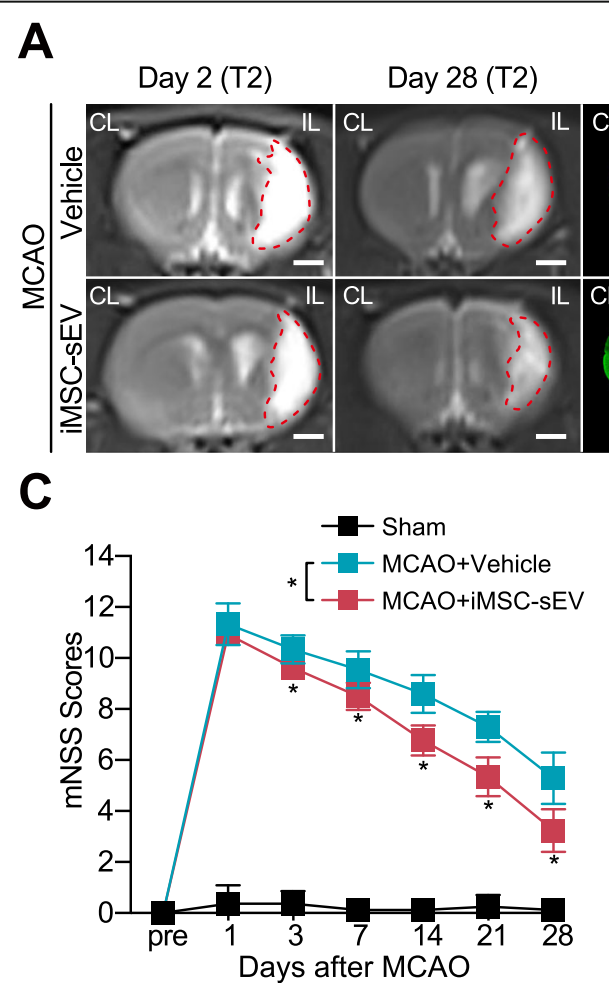

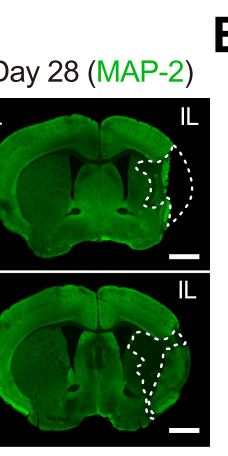

B $\square \mathrm{MCAO}+\mathrm{Vehicle}$

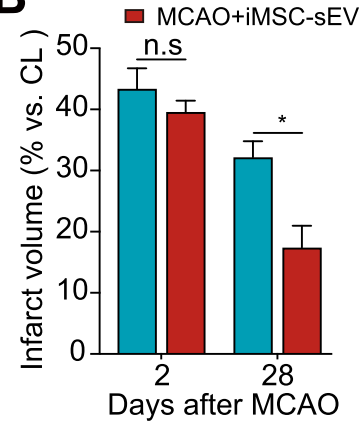

D

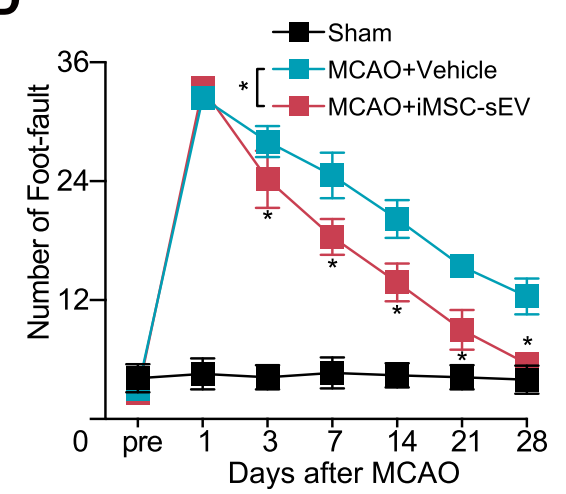

Fig. 2 iMSC-sEV treatment reduces tissue loss in the chronic stage of ischemic stroke and promotes long-term neurological recovery. a Representative images of T2-MRI scan (left two columns) performed on days 2 and 28, as well as MAP-2 immunostaining (Right column) on day 28 after MCAO. Dashed line (red and white): infarct border. CL, contralateral side. IL, ipsilateral side. Scale bar $=2 \mathrm{~mm}$. $\mathbf{b}$ Quantification analysis of the percentage of infarct volume on day 2 and day 28 after MCAO. $N=8$ rats per group. Behavior tests were performed by using mNSS score (c) and foot-fault test (d) before and up to 28 days after MCAO. $N=5$ rats for sham group. $N=10$ rats for vehicle and iMSC-sEV groups. Data are presented as mean $\pm S D$. ${ }^{*} P<0.05$. n.s indicates no significant difference 
after MCAO (Fig. 2a, b). Measurements from behavioral tests were applied to examine whether iMSC-sEV contribute to long-term improvement in neurological function after stroke. As expected, compared with vehicle treatment, iMSC-sEV significantly enhanced functional recovery, as reflected by significantly decreased mNSS (Fig. 2c) and lower error step number in the foot-fault test (Fig. 2d), starting from early 3 days to 28 days after stroke. These results indicated that iMSC-sEV improve functional outcomes in rats after cerebral ischemic injury.

\section{iMSC-sEV enhance angiogenesis after ischemic stroke}

Angiogenesis is positively correlated with neurological function recovery after stroke [6]. Next, we examined if iMSC-sEV could promote angiogenesis after ischemic stroke in rats. Newly formed vessels and mature vessels in the infarct boundary zone were characterized by CD31/EdU, CD34 staining, and CD31 staining [38]. The vehicle group induced a significantly higher expression of $\mathrm{CD}^{+} 1^{+} / \mathrm{EdU}^{+}$(Fig. 3a, b) and CD34 ${ }^{+}$endothelial cells (Fig. 3c, d) than the sham group, suggesting a selfprotecting mechanism of spontaneous angiogenesis after stroke insult. Notably, a further increased number of $\mathrm{CD}^{+} 1^{+} / \mathrm{EdU}^{+}$(Fig. 3a, b) and $\mathrm{CD}^{+}$cells (Fig. 3c, d) were detected in the iMSC-sEV group as compared to the vehicle group. In addition, comparing to PBS-treated group, the overall blood vessel density was increased in the iMSC-sEV treatment group 7 days after MCAO (Fig. S3A-B). Taken together, these data suggested that poststroke iMSC-sEV administration promotes angiogenesis.

\section{iMSC-sEV promote migration and tube formation of HUVECs subjected to OGD}

To further determine the effect of iMSC-sEV on the function of endothelial cells, HUVECs were applied to oxygen and glucose deprivation (OGD), a wellestablished in vitro model to mimic ischemic stroke. First of all, HUVECs were identified by the expression of CD31 (Fig. S4A) and vWF (Fig. S4B) but not $\alpha-S M A$ (Fig. S4C). We then determined whether iMSC-sEV could be internalized by HUVECs. iMSC-sEV were labeled with Dil fluorescent dye and added to HUVECs culture medium. After $4 \mathrm{~h}$ of incubation, Dil-labeled iMSC-sEV were efficiently up-taken by HUVECs (Fig. S4D). HUVECs subjected to OGD exhibited significantly decreased abilities of migration and tube formation as compared to control group, as shown in Fig. $3 \mathrm{e}-\mathrm{h}$ iMSC-sEV significantly enhanced the migration capability of HUVECs when compared to the vehicle treatment. Besides, an improved tube forming ability was also visualized in the iMSC-sEV group, compared to that in the vehicle group $24 \mathrm{~h}$ after administration. Collectively, these data indicated that iMSC-sEV promote migration and tube formation of HUVECs subjected to OGD in vitro.

\section{iMSC-sEV alleviate ischemic stroke-induced autophagy in vivo and in vitro}

Autophagy occurred in endothelial cells in a rat model of MCAO [28]. We then investigated the relationship between autophagy and the pro-angiogenic effects of iMSC-sEV in ischemic stroke models in vivo and in vitro. We first detected the autophagy-associated protein levels after MCAO by western blot. The results showed that the expression of LC3-II/LC3-I and Beclin1 was significantly increased while P62 protein level was decreased after stroke model (Fig. 4a, b). However, iMSC-sEV markedly reduced the protein levels of LC3II/LC3-I and Beclin-1 and increased P62 level. We also used TEM to detect autophagy in vivo. The specific autophagosomes which is characterized by doublemembrane structure was visualized in the vehicle group but not the sham group (Fig. 4c). Notably, the iMSCsEV group showed less autophagosome.

Next, to detect autophagy in endothelial cells after stroke, HUVECs were subjected to an 8-h OGD. In consistent with the in vivo data, results from western blot demonstrated that OGD increased levels of LC3-II/LC3$\mathrm{I}$ and Beclin-1 while decreased p62 protein levels in HUVECs compared to control group, and iMSC-sEV dramatically suppressed OGD-induced LC3-II/LC3-I and Beclin-1 expression and increased p62 expression in HUVECs (Fig. 4d, e). TEM indicated that iMSC-sEV reduced the formation of autophagosomes during OGD in HUVECs (Fig. 4f). These results indicated that iMSCsEV inhibit ischemic stroke-triggered autophagy.

We have proved iMSC-sEV treatment promoted angiogenesis and inhibited autophagy in endothelial cells after stroke. To further prove the interaction between autophagy and angiogenesis in stroke conditions, 3methyladenine (3-MA), a well-known autophagy inhibitor, was applied. As expected, 3-MA reversed the decline of migration (Fig. S5A-B) and tube formation (Fig. S5CD) induced by OGD in HUVECs, suggesting that increase of angiogenesis is related to the inhibition of autophagy in HUVECs after stroke.

\section{iMSC-sEV activated STAT3 signaling pathway in vivo and in vitro}

Many signaling pathways mediate autophagy, and STAT3 has been considered as a classical inhibiting factor [39]. Therefore, STAT3 was detected in vivo and in vitro using western blot analysis. Phosphorylated STAT3 decreased significantly in ischemic stroke rats compared to sham rats, and iMSC-sEV dramatically reversed this decrease (Fig. 5a). A similar result was confirmed in vitro (Fig. 5b). Altogether, these results 

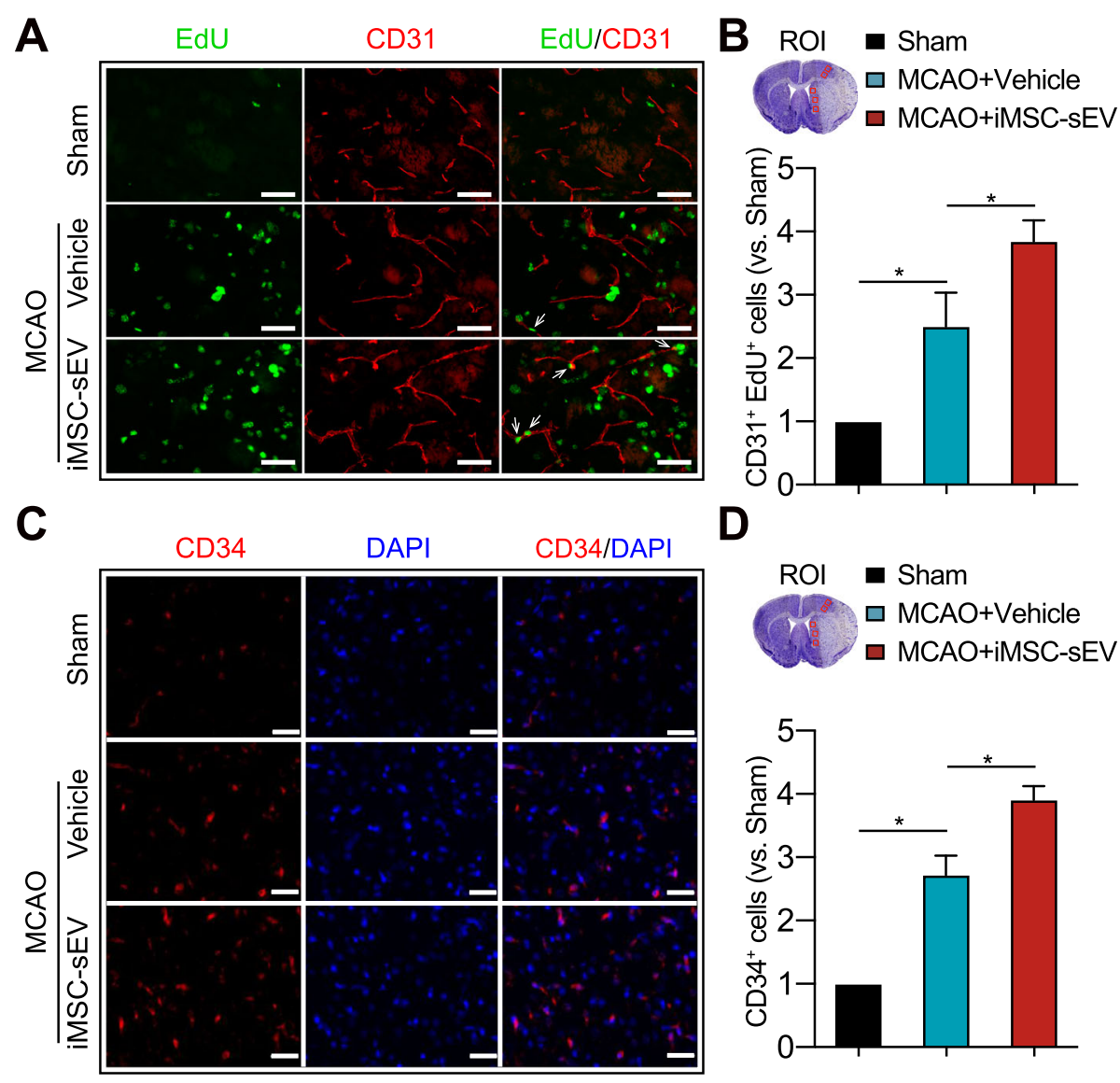

D

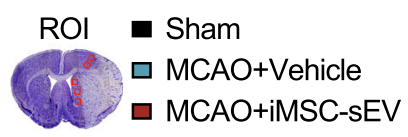

E
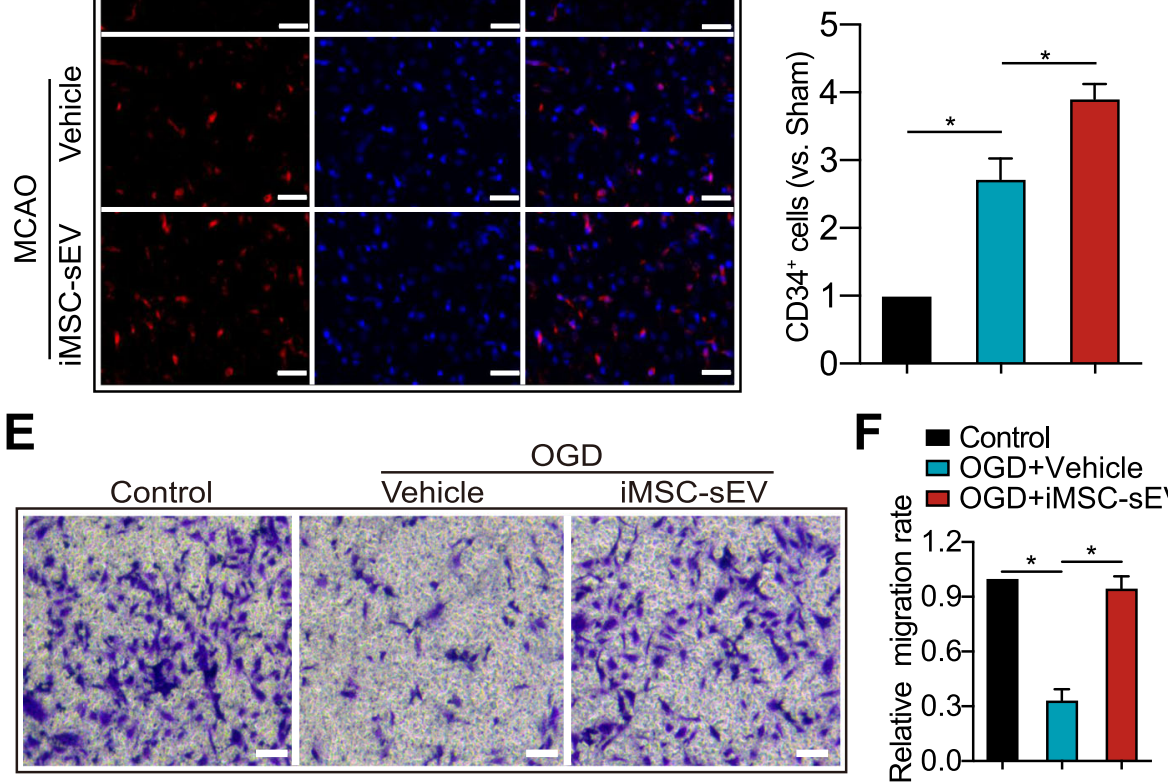

G

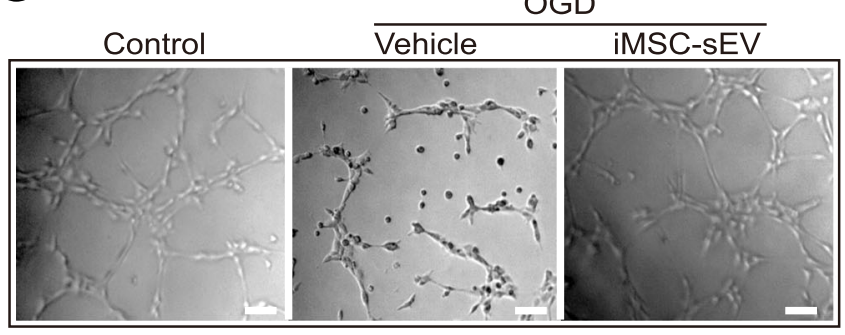

F Control

$\square$ OGD+Vehicle

๑ OGD+iMSC-sEV

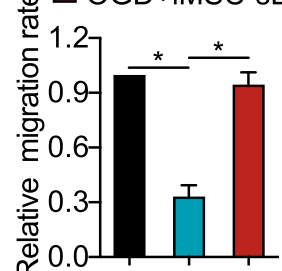

H

$\square$ OGD+Vehicle

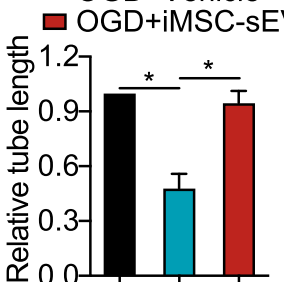

Fig. 3 (See legend on next page.) 


\section{(See figure on previous page.)}

Fig. 3 iMSC-sEV promote angiogenesis after stroke and increase migration and tube formation in HUVECs after OGD. a-d Angiogenesis was assessed by immunofluorescence staining of CD31/EdU and CD34 at 7 days after MCAO. a Representative images of CD31 (red) and EdU (Green) in the ischemic boundary zone. Arrow indicates $\mathrm{CD}_{3} 1^{+} \mathrm{EdU} U^{+}$proliferated endothelial cells. Scale bar $=100 \mu \mathrm{m}$. b Nissl staining (upper left) showing the region of interest (ROI) and quantification analysis of $\mathrm{CD} 31^{+} \mathrm{EdU} \mathrm{U}^{+}$cells normalized to that in sham group. Red box: ROI of CD31/EdU staining. $N=3-5$ per group. c Representative images of CD34 (red) and DAPI (blue) in the peri-infarct area. Scale bar $=100 \mu$ m. $\mathbf{d}$ Nissl staining (upper left) showing $\mathrm{ROI}$ of the staining and quantification analysis of $\mathrm{CD} 34^{+}$cells normalized to that in sham group. Red box: $\mathrm{ROI}$ of CD34 staining. $N=3-5$ per group. e-h HUVECS were challenged with $8 \mathrm{~h}$ OGD, followed by iMSC-sEV or vehicle treatment for $24 \mathrm{~h}$. HUVECs cultured under the normoxia condition without treatment were set as control. e Representative images of crystal violet staining in the transwell assay. Scale bar $=25 \mu \mathrm{m} . \mathbf{f}$ Quantification analysis of migration rate normalized to that in control group. $N=3$ per group. $\mathbf{g}$ Representative images of the tube formation assay. Scale bar $=25 \mu \mathrm{m}$. $\mathbf{h}$ Quantification analysis of the relative tube length normalized to that in control group. $N=3$ per group. Data are presented as mean \pm SD. ${ }^{*} P<0.05$

suggested that iMSC-sEV activate the STAT3 signaling pathway.

\section{STAT3 signaling pathway is involved in iMSC-sEV's inhibition of stroke-induced autophagy}

iMSC-sEV significantly activated STAT3 after ischemic stroke in vivo and in vitro. We next used stattic, a STAT3 inhibitor, to further confirm the role of STAT3 in the preventative effects of iMSC-sEV on strokeinduced autophagy. Western blot analysis showed that stattic significantly decreased p-STAT3/STAT3 ratio in the iMSC-sEV supplemented with stattic group as compared to iMSC-sEV group both in vivo (Fig. 6a, b) and in vitro (Fig. 6c, d), suggesting stattic inhibited iMSCsEV-induced STAT3 activation. Accompanied by this phenomenon, stattic also abolished the iMSC-sEVinduced reduction of Beclin-1and LC3-II/LC3-I, and increment of p62 both in vivo (Fig. 6a, b) and in vitro (Fig. $6 c, d)$. These results suggested that the STAT3 signaling pathway takes part in iMSC-sEV's suppression of strokeinduced autophagy after stroke.

\section{iMSC-sEV facilitate angiogenesis partially via upregulation of STAT3 in vivo and in vitro}

The STAT3 inhibitor stattic was used to examine whether the pro-angiogenic effect of iMSC-sEV on ischemic stroke was related to STAT3-dependent autophagy. Stattic significantly abolished the iMSC-sEVinduced increase of $\mathrm{CD}^{+} 4^{+}$new-born endothelial cells (Fig. 7a, b), CD $31^{+} E d U^{+}$proliferated endothelial cells (Fig. S6A-B), and CD31 ${ }^{+}$vessel density (Fig. S6C-6D) in the infarct boundary zone 7 days after MCAO. However, the diminishment induced by STAT3 blocking did not reach to the level of vehicle group, indicating another pathway may be involved in the promotion effect of iMSC-sEV on angiogenesis (Fig. 7a, b). Besides, in vitro study also showed that stattic reversed the promotion of migration and tube formation by iMSC-sEV in HUVECs after OGD (Fig. 7c-f). Taken together, these data indicated that STAT3 play an important role in the proangiogenic effect by iMSC-sEV in ischemic stroke.

\section{Discussion}

In this study, we found that iMSC-sEV reduced infarction size and improved neurological recovery in an experimental ischemic stroke model in rats. We further demonstrated that iMSC-sEV significantly increased newly formed blood vessels and mature vessels after stroke. Moreover, iMSC-sEV promoted both migration and tube formation in HUVECs subjected to OGD. Mechanistically, iMSC-sEV promoted angiogenesis in the ischemic brain, in part, via suppression of autophagy, a process that is dependent on STAT3 activation. The present study is the first to report that iMSC-sEV promote angiogenesis and protect against ischemic brain injury, potentially, via the inhibition of autophagy, and STAT3 pathway played an important role in this process.

Angiogenesis is a complicated and sequential process which plays a crucial role in ischemic brain injury. The newly formed vessels after stroke not only improved tissue perfusion but also closely linked with neurovascular remodeling [40], axonal sprouting [41], and remyelination [42]. It is well established that angiogenesis is strongly associated with improvement of neurological deficits after stroke $[5,6]$. Patients with higher blood vessel density showed better functional recovery after ischemic stroke impact. The ability to promote angiogenesis in MSC from different sources, for example, bone marrow [43], human umbilical blood [44], and adipose [45], were widely studied. However, current methods for the large-scale preparation of MSC face several challenges as the amount of MSC that may be obtained from donors is often insufficient. Furthermore, the potential of growth and differentiation in vitro is affected by various factors such as culture period, age, and health condition of the donor. Given that iMSC provide an ideal method that can avoid ethical problem and immune rejection, our strategy for sEV production offers several advantages considering the limitations related to the present applications of MSC. Previous studies from our group demonstrated that iMSC-sEV had a strong therapeutic function by promoting angiogenesis in different disease models including limb ischemia [16] and 


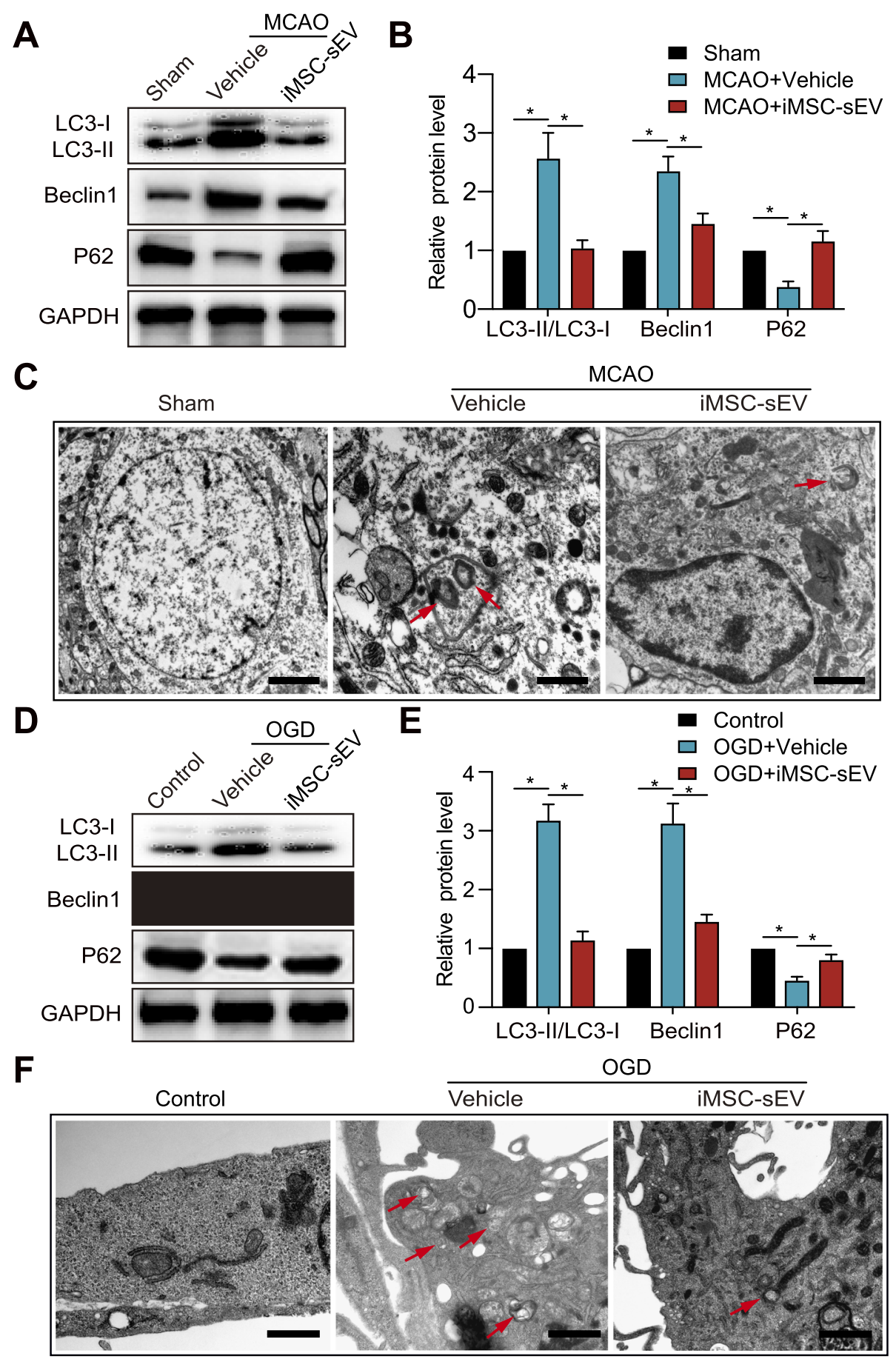

Fig. 4 iMSC-sEV treatment alleviates stroke-induced autophagy in vivo and in vitro. Ipsilateral infarct brain tissue was harvested $24 \mathrm{~h}$ after MCAO or Sham operation for western blotting analysis $(\mathbf{a}-\mathbf{b})$ or TEM observation (c). $\mathbf{a}$, b Protein expression levels of autophagy associated proteins including LC3-I/LC3-I, Beclin-1, and P62 in the indicated groups. N=3-5 per group. c Representative images of TEM showing autophagy-related morphological change after MCAO. Red arrow: autophagosome. Scale bar $=1 \mu \mathrm{m}$. $\mathbf{d}-\mathbf{f}$ HUVECs were cultured in OGD condition for $8 \mathrm{~h}$, followed by normoxia condition with the treatment of iMSC-sEV or vehicle for another $24 \mathrm{~h}$. HUVECs cultured under normoxia condition without treatment were used as control. d, e Western blotting was used to evaluate LC3-II/LC3-I, Beclin-1, and P62 protein levels in HUVECs. $N=3$ per group. $\mathbf{f}$ Representative TEM images of HUVECs with or without OGD challenge. Red arrow: autophagosome. Scale bar $=1 \mu \mathrm{m}$. Data are presented as mean \pm SD. ${ }^{*} P<0.05$ 


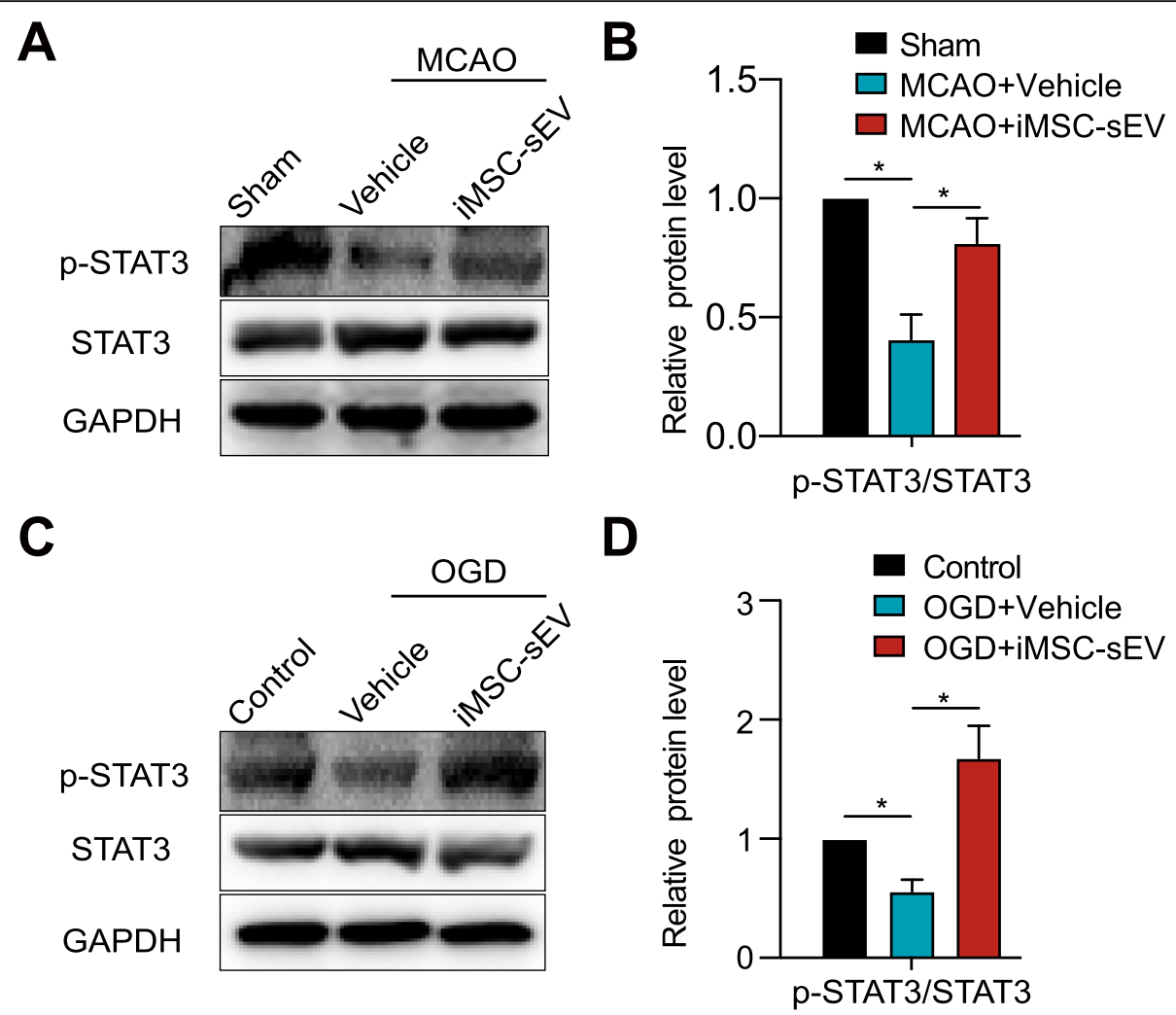

Fig. 5 iMSC-sEV activate STAT3 signaling pathway in vivo and in vitro. $\mathbf{a}$, b Western blotting analysis showing the expression level of p-STAT3 and STAT3 in the ipsilateral infarct brain $24 \mathrm{~h}$ after MCAO or sham operation. $\mathrm{N}=3-5$ per group. $\mathbf{c}, \mathbf{d}$ HUVECs were cultured in OGD condition for $8 \mathrm{~h}$, followed by normoxia condition with the treatment of iMSC-sEV or vehicle for another $24 \mathrm{~h}$. Western blotting analysis was performed to evaluate the expression of p-STAT3 and STAT3 in HUVECS in indicated groups. $N=3$ per group. Data are presented as mean \pm SD. ${ }^{* P}<0.05$

osteonecrosis [46]. Thus, we proposed that iMSC-sEV may also have a similar function in ischemic stroke. Consistent with our hypothesis, rats treated with iMSCsEV presented with decreased brain tissue loss, improved neurological outcome, and higher vessel density after stroke, suggesting that iMSC-sEV attenuate ischemic brain injury partially through promotion of angiogenesis. We further found that in vitro ischemia model, OGD, induced markedly decline in endothelial functions (migration and tube formation) which was rescued by iMSC-sEV treatment, confirming the ability of iMSCsEV in promoting angiogenesis under stroke conditions.

Autophagy is activated after brain ischemia, and it is detected in different brain cells including neurons, oligodendrocytes, endothelial cells, and so on ${ }^{26}$. The effect of autophagy on ischemic injury remains controversial with some studies reported a protective role while others showed a deleterious function [47]. For example, activation of autophagy by rapamycin, a mTOR inhibitor, reduced infarction and improved outcome in murine models of MCAO [48]. In contrast, Shi et al. reported that excessive autophagy contributes to neuronal death [27]. Additionally, reducing autophagy both in vitro and in vivo is beneficial during ischemic stroke $[49,50]$. In our study, we found that autophagy is markedly activated in infarct penumbra $24 \mathrm{~h}$ after stroke or in OGDtreated HUVECs. iMSC-sEV administration downregulated the protein expression of Beclin1 and the LC3-II/I ratio, both of which are markers for autophagy, and upregulated the expression of $\mathrm{p} 62$ in vivo and in vitro. The controversial action of autophagy in ischemic stroke studies may be due to the complexity of experimental settings including differences in animal model and stage and intensity of ischemia [51]. Indeed, we conducted a 2-h MCAO model in rats, which maybe induced excessive and prolonged autophagy which is detrimental after stroke. Previous studies reported a complicated crosslink between angiogenesis and autophagy. Some studies have shown that cellular autophagy may enhance angiogenesis in endothelial cells $[52,53]$. On the contrary, increasing evidence suggested that cellular autophagy can inhibit the angiogenesis in endothelial cells [54-56]. Consistent with previous studies which demonstrated that MSCsEV can regulate autophagy [57], our results showed that ischemia-induced autophagy suppressed angiogenesis in vivo and in vitro, while inhibition of autophagy by iMSC-sEV significantly promoted angiogenesis in vivo and facilitated cell migration and tube formation in 


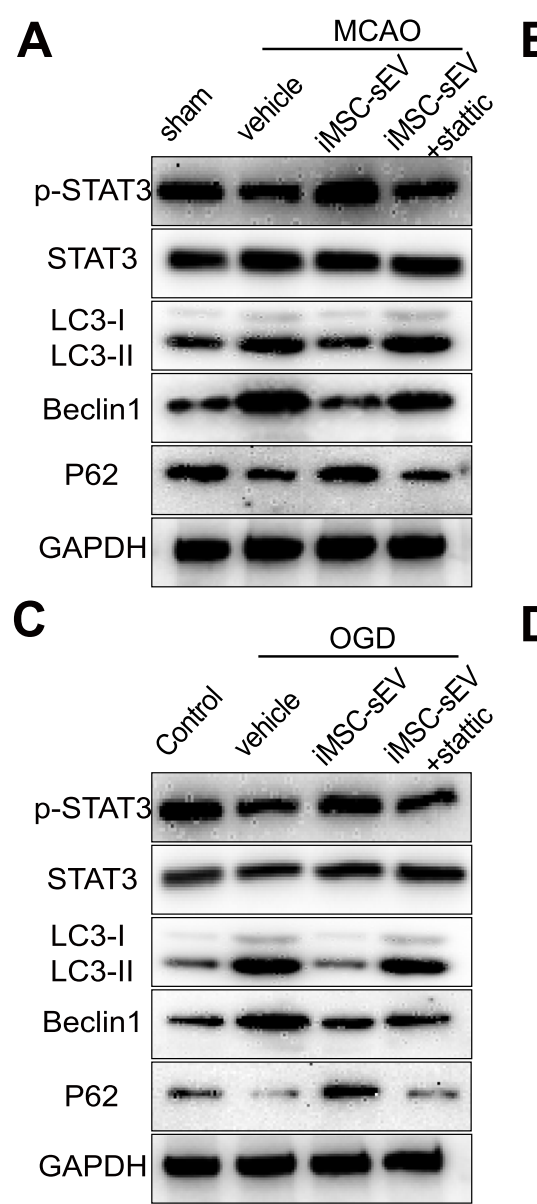

B

$$
\begin{aligned}
& \text { Sham } \\
& \square \text { MCAO+Vehicle } \square \text { MCAO+iMSC-sEV+stattic }
\end{aligned}
$$

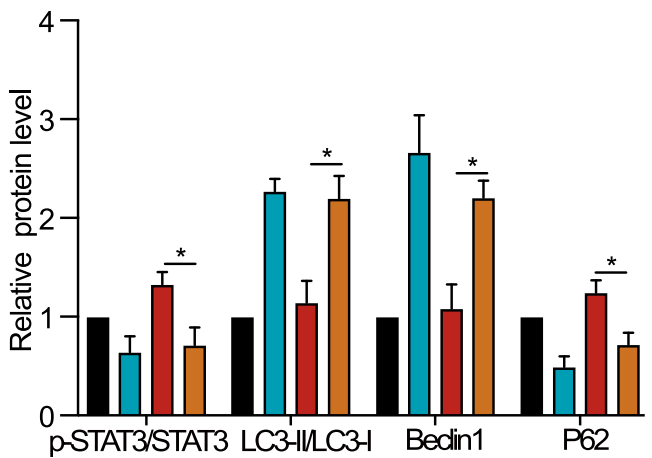

D

$$
\begin{array}{ll}
\text { Control } & \square \text { OGD+iMSC-sEV } \\
\square \text { OGD+Vehicle } & \square \text { OGD+iMSC-sEV+stattic }
\end{array}
$$

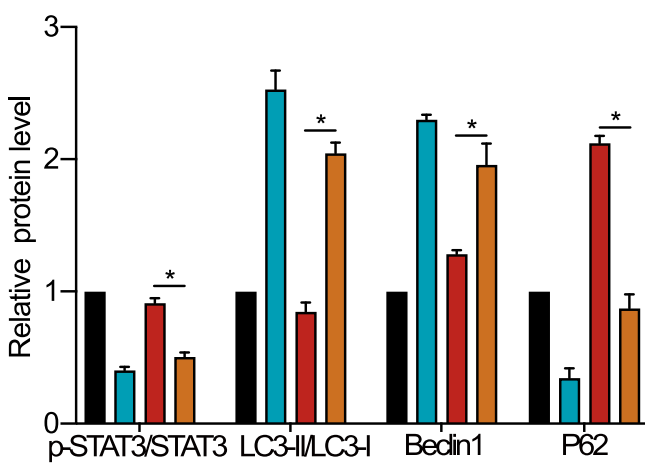

Fig. 6 iMSC-sEV inhibit stroke-induced autophagy partially via activation of STAT3 signaling pathway. $\mathbf{a}$, b Rats were intravenously treated with vehicle $(500 \mu \mathrm{l}, \mathrm{PBS})$, iMSC-sEV $\left(1 \times 10^{11}\right.$ particles in $500 \mu \mathrm{L}$ PBS), or iMSC-sEV with stattic $(3.75 \mathrm{mg} / \mathrm{kg}) 4 \mathrm{~h}$ after MCAO, and infarct brain tissue from different groups was harvested $24 \mathrm{~h}$ after MCAO for western blotting. Sham rats were used as control. Representative images (a) and quantification (b) of the proteins including p-STAT3/STAT3, LC3-II/LC3-I, Beclin-1, and P62 in the indicated groups. N=3-5 per group. c, d HUVECS were challenged by OGD for $8 \mathrm{~h}$, followed by treatment with vehicle (PBS), iMSC-sEV $\left(1 \times 10^{9}\right.$ particles $\left./ \mathrm{mL}\right)$, or iMSC-sEV with stattic $(5 \mu \mathrm{M})$ under normal culture condition for another $24 \mathrm{~h}$. HUVECs cultured under normoxia condition without treatment were used as control. Representative bands (c) and quantification (d) of the proteins including p-STAT3/STAT3, LC3-II/LC3-I, Beclin-1, and P62 in the indicated groups. N=3 per group. Data are presented as mean \pm SD. ${ }^{*} P<0.05$

OGD-treated HUVECs in vitro. These data suggested that iMSC-sEV may increase angiogenesis by inhibiting endothelial autophagy during the recovery of ischemic stroke.

The reasons underlying the inhibitory effect of MSCsEV against autophagy under ischemic conditions may be involved with the transfer of substances contained in sEV including microRNAs [58-61] and functional proteins [62]. For example, miR-20a containing sEV derived from umbilical cord mesenchymal stem cells (UC-MSCs) inhibited autophagy by binding to 3' UTR of Beclin-I and alleviated liver ischemia/reperfusion injury [59]. In addition, in an animal model of myocardial infarction (MI), treatment with MSC-derived sEV overexpressing miR-125b reduced autophagic flux and infarct size, along with improved cardio functions [61]. Upregulation of stromal-derived factor 1 (SDF1a) with SDF1 plasmid in MSC-derived sEV inhibited ischemia-induced autophagy and promoted cardiac endothelial microvascular regeneration after MI insult [62].

Many signaling pathways mediate autophagy, and STAT3 is a classical inhibiting factor [39]. The present study detected that ischemic stroke decreased STAT3 activation in vivo and in vitro. iMSC-sEV significantly upregulated the STAT3 signaling pathway in the periinfarct area in rats after stroke and cultured HUVECs subjected to OGD in the present study. Moreover, blocking STAT3 activation partially abolished iMSCsEV's inhibition of stroke-induced autophagy and angiogenesis. Previous studies demonstrated that STAT3 reduced Beclin-1 expression, thus suppressing autophagy via inhibition of oxidative stress and autophagy-related 


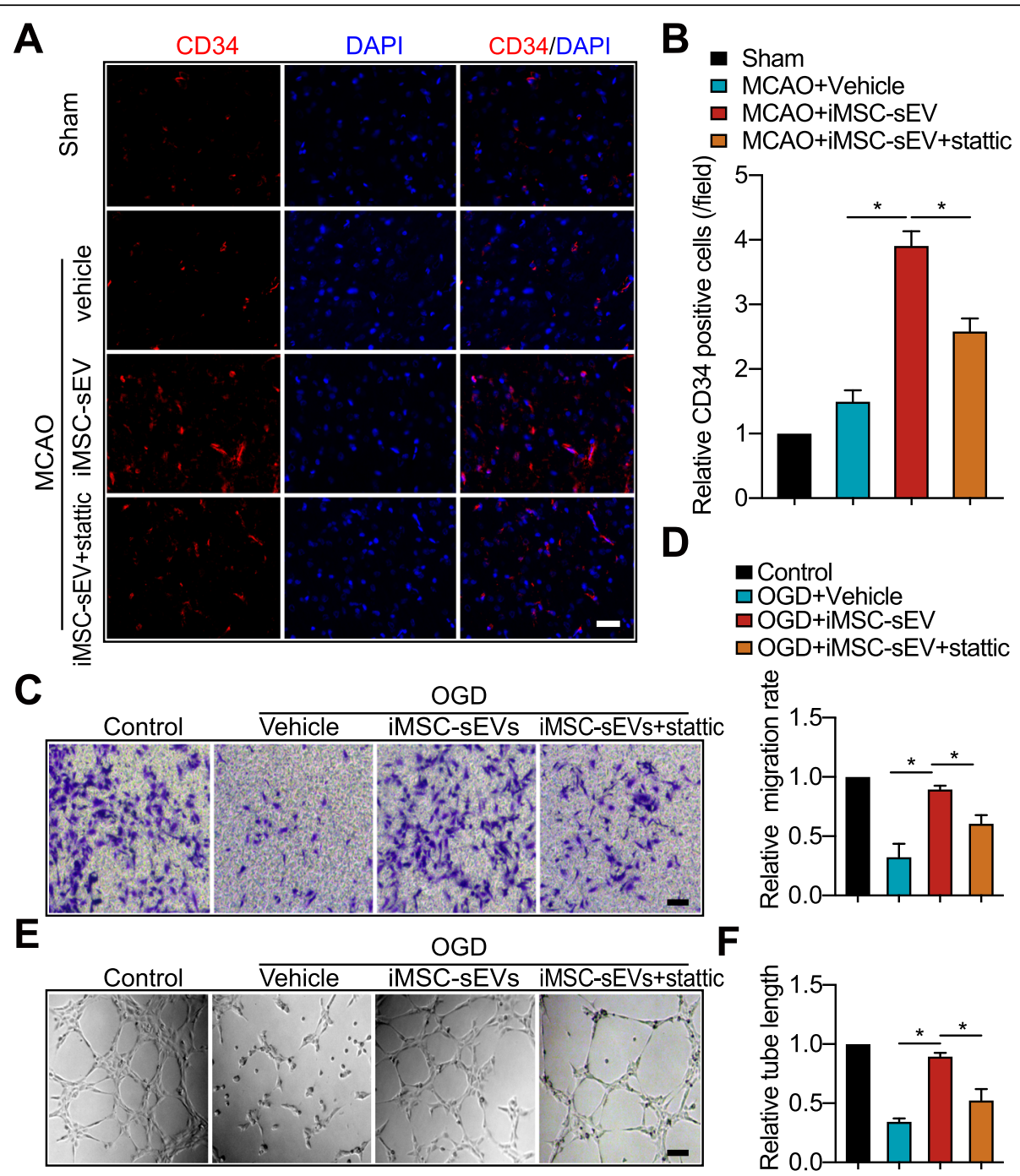

Fig. 7 Inactivation of STAT3, partly, inhibits the pro-angiogenic effect of iMSC-sEV after the experimental stroke model. $\mathbf{a}$, $\mathbf{b}$ Rats were intravenously treated with vehicle $(500 \mu \mathrm{l}, \mathrm{PBS})$, iMSC-sEV $\left(1 \times 10^{11}\right.$ particles in $\left.500 \mu \mathrm{L} \mathrm{PBS}\right)$, or iMSC-sEV with stattic $(3.75 \mathrm{mg} / \mathrm{kg}) 4 \mathrm{~h}$ after MCAO; angiogenic ability was assessed 7 days after MCAO or Sham surgery. a Representative images of immunofluorescence staining of CD34 in the peri-infarct area. Scale bar $=100 \mu \mathrm{m}$. b Quantification analysis of CD34 ${ }^{+}$cells normalized to that in sham group. $N=3$ per group. $\mathbf{c}-\mathbf{f}$ HUVECs were challenged by OGD for $8 \mathrm{~h}$, followed by treatment with vehicle (PBS), iMSC-sEV $\left(1 \times 10^{9}\right.$ particles $\left./ \mathrm{mL}\right)$, or iMSC-sEV with stattic $(5 \mu \mathrm{M})$ in normal culture medium for another $24 \mathrm{~h}$. HUVECs cultured under normoxia condition without any treatment were deemed as control. c Representative images of crystal violet staining of HUVECS in the transwell assay. Scale bar $=25 \mu \mathrm{m}$. $\mathbf{d}$ Quantification analysis of relative migration rate. $N=3$ per group. e Representative images of the tube formation assay. Scale bar $=5 \mu \mathrm{m}$. $\mathbf{f}$ Quantification analysis of relative tube length in the indicated groups. $N=3$ per group. Data are presented as mean \pm SD. ${ }^{*} P<0.05$

signaling molecules such as FOXO1 and FOXO3 [39]. Therefore, STAT3 may inhibit autophagy via a reduction in Beclin-1 in ischemic stroke. The results of the present study demonstrated that iMSC-sEV increased STAT3 activation during ischemic stroke, which was accompanied by a reduction in Beclin-1. However, STAT3 inhibitor abolished the iMSC-sEV-induced inhibition of Beclin-1. These results indicated that iMSC-sEV inhibits ischemic stroke-provoked autophagy via a STAT3dependent pathway. The role of STAT3 signaling pathway on brain vessel after stroke is multifaceted.
Conditional knockout of endothelial STAT3 reduced angiogenesis and exacerbated neurological deficits after stroke, suggesting an important role of STAT3 in regulating angiogenesis [63]. This result is consistent with our study where increased activation of STAT3 by iMSC-sEV promoted angiogenesis and inactivation of STAT3 by stattic partially attenuated this effect, indicating a potential STAT3-involved mechanism in iMSCsEV-induced pro-angiogenic ability after ischemic brain injury, while, a recent paper reported that inhibition of STAT3 by stattic improved blood brain barrier (BBB) 
integrity after stroke [64]. Indeed, the effect of STAT3 activation on other aspects such as neuronal death and neuroinflammation after stroke is still in debate [65]. Further investigation to elucidate the thorough functions of STAT3 after stroke is in need.

\section{Conclusion}

In summary, the present study reported, for the first time, that iMSC-sEV treatment protects against ischemic injury and promotes angiogenesis probably via the inhibition of autophagy. The mechanism for iMSC-sEV's inhibition of autophagy was partially due to the activation of STAT3. This finding provides a novel treatment strategy for ischemic stroke and uncovers new molecular mechanisms underlying the application of iMSC-sEV.

\section{Supplementary information}

Supplementary information accompanies this paper at https://doi.org/10. 1186/s13287-020-01834-0.

Additional file 1: Figure S1. Phenotypic characteristics of iMSC. (A) Flow cytometry analysis of the surface antigen profile of iMSC. (B-D) Representative images of Alizarin Red staining (B), Oil Red O staining (C), and Toluidine Blue staining (D) for the evaluation of osteogenesis, adipogenesis, and chondrogenesis in iMSC. Scale bar $=25 \mu \mathrm{m}$.

Additional file 2: Figure S2. In vivo uptake of iMSC-sEV after stroke. (A) One single dose of vehicle (PBS, $500 \mu \mathrm{L}$ ) or DiR labeled iMSC-sEV (DiRiMSC-sEV, $1 \times 10^{11}$ particles in $500 \mu \mathrm{L}$ PBS) were administered through tail veil injection in rats $4 \mathrm{~h}$ after MCAO and images were captured $6 \mathrm{~h}$ after administration. Representative fluorescence images of rats brain in the vehicle and DiR-iMSC-sEV group. IL: ipsilateral side. CL: contralateral side. (BC) Dio labeled iMSC-sEV (Dio-iMSC-sEV) were administered intravenously $24 \mathrm{~h}$ after MCAO, and rats were sacrificed $24 \mathrm{~h}$ after injection. (B) Illustration of experimental design. (C) Representative images of Dio-iMSC-sEV (green) in the ipsilateral and contralateral side of the brain. Yellow dashed line: outline for blood vessel. Red arrow head: Dio-iMSC-sEV around the nucleus in the cytoplasm. Scale bar $=50 \mu \mathrm{m}$.

Additional file 3: Figure S3. Treatment of iMSC-sEV increases the blood vessel density after ischemic stroke. CD31 immunofluorescence staining was utilized to evaluate blood vessel density 7 days after MCAO. (A) Representative images of CD31 positive endothelial cells and DAPI staining in the peri-infarct area. Scale bar $=400 \mu \mathrm{m}$. (B) ROI and quantification of $\mathrm{CD} 1^{+}$blood vessel density. $N=3-5$ per group. Data are presented as mean \pm SD. ${ }^{*} \mathrm{P}<0.05$.

Additional file 4: Figure S4. Identification of HUVECs and in vitro uptake of iMSC-sEV by HUVECS. (A-C) Representative immunofluorescence images of CD31 (green), vWF (green), and a-SMA (red) in HUVECs. Scale bar $=100 \mu \mathrm{m}$. (D) Representative immunofluorescence images of HUVECs cultured with Dil labeled iMSC-sEV (red) or Dil alone (control). The Dil-labeled iMSC-sEV were visible in the perinuclear region of recipient cells. HUVECs in the control group showed no fluorescence signal. Scale bar $=30 \mu \mathrm{m}$.

Additional file 5: Figure S5. Inhibition of autophagy decreases migration and tube formation in HUVECs after OGD. HUVECs were challenged with $8 \mathrm{~h}$ OGD with the addition of 3-MA (5 mM) or vehicle (PBS) and migration and tube formation were analyzed $24 \mathrm{~h}$ after reoxygenation. HUVECs cultured under the normoxia condition without treatment were set as control. (A) Representative images of crystal violet staining in the transwell assay. Scale bar $=25 \mu \mathrm{m}$. (B) Quantification analysis of migration rate normalized to control group. $\mathrm{N}=3-5$ per group. (G) Representative images of the tube formation assay. Scale bar = $25 \mu \mathrm{m}$. (H) Quantification analysis of the tube length normalized to control group. $\mathrm{N}=3-5$ per group. Data are presented as mean $\pm \mathrm{SD}$. ${ }^{*} \mathrm{P}<0.05$.
Additional file 6: Figure S6. Stat3 inactivation inhibites iMSC-sEVinduced angiogenesis. (A-D) Rats were intravenously treated with vehicle (500ul, PBS), iMSC-sEV $\left(1 \times 10^{11}\right.$ particles in $500 \mu \mathrm{L}$ PBS), or iMSC-sEV with stattic $(3.75 \mathrm{mg} / \mathrm{kg}) 4 \mathrm{~h}$ after MCAO, EdU was i.p. injected at day 3, 5, and 7 to label proliferated cells. (A) Representative images of $\mathrm{CD} 31^{+} \mathrm{EdU}^{+}$ endothelial cells in the peri-infarct area. Arrow head, double-labeled proliferated cells. Scale bar $=100 \mu \mathrm{m}$. (B) Quantification of $\mathrm{CD}_{3} 1^{+} \mathrm{EdU} \mathrm{U}^{+}$endothelial cells normalized to that in sham group. $\mathrm{N}=3-5$ per group. (C) Representative of $\mathrm{CD}_{3} 1^{+} \mathrm{DAPI}^{+}$endothelial cells in the peri-infarct zone. Scale bar $=400 \mu \mathrm{m}$. (D) Quantification of $\mathrm{CD}_{3} 1^{+}$vascular density normalized to that in Sham group. $\mathrm{N}=3-5$ per group.

\section{Abbreviations}

iPSC: Human induced pluripotent stem cells; MSC: Mesenchymal stem cells; STAT3: Signal transducer and activator of transcription 3; sEV: Small extracellular vesicles; MCAO: Middle cerebral artery occlusion; iMSC-sEV: Small extracellular vesicles secreted by mesenchymal stem cell derived from human induced pluripotent stem cells; tPA: Tissue plasminogen activator; ESC: Embryonic stem cells; NSC/NPC: Neural stem/precursor cells; BBB: Bloodbrain barrier; OGD: Oxygen and glucose deprivation; HUVECs: Human umbilical vein endothelial cells; MV: Microvesicles; PBS: Phosphate Buffer Saline; TEM: Transmission electron microscopy; SD: Sprague Dawley; MAP2: Microtubule associated protein 2; MRI: Magnetic resonance imaging; mNSS: Modified neurological severity score; FBS: Fetal bovine serum; RO: Reoxygenation; ANOVA: One-way analysis of variance; SDF1a: Stromalderived factor 1; USC-sEV: Urinal stem cell derived sEV; MI: Myocardial infarction

\section{Acknowledgements}

Not applicable.

\section{Authors' contributions}

Y.X.: conception and design, collection and assembly of data, data analysis and interpretation, and manuscript writing; X.L.: collection and assembly of data, data analysis and interpretation, and manuscript writing; Q.Z. and J.Z. collection of data; H.W. and Q.L. data analysis and interpretation; B.Z. financial support; Z.D. and Y.W.: conception and design, financial support, provision of study material, data analysis and interpretation, and final approval of manuscript. The authors read and approved the final manuscript.

\section{Funding}

This work was supported by the National Natural Science Foundation of China (Grant No. 81671209, 81870972, and 81871833).

\section{Availability of data and materials}

The datasets used and/or analyzed during this study are available from the corresponding author on reasonable request.

\section{Ethics approval and consent to participate}

The use of human iPSC in this study was approved by the local ethics committee of the Shanghai Sixth People's Hospital affiliated to Shanghai Jiao Tong University. HUVECs were obtained with informed consent and approval by the local ethics committee of the Shanghai Sixth People's Hospital affiliated to Shanghai Jiao Tong University. This work was conducted in compliance with the principles of the Helsinki Declaration and Guidelines for the Care and Use of Laboratory Animals of the Chinese Institute of Health. All of the animal study protocols were approved by the Animal Research Committee of the Shanghai Sixth People's Hospital (SYXK [Shanghai, China] 2011-0128, 1 January 2011), and all of the animal-related procedures were performed in compliance with the approved guidelines.

\section{Consent for publication}

Not applicable.

\section{Competing interests}

The authors indicated no potential conflicts of interest. 
Received: 6 February 2020 Revised: 21 June 2020

Accepted: 14 July 2020 Published online: 22 July 2020

\section{References}

1. Collaborators GBDS. Global, regional, and national burden of stroke, 19902016: a systematic analysis for the global burden of disease study 2016. Lancet Neurol. 2019;18:439-58.

2. Linfante I, Cipolla MJ. Improving reperfusion therapies in the era of mechanical thrombectomy. Transl Stroke Res. 2016;7:294-302.

3. Benjamin EJ, Muntner P, Alonso A, Bittencourt MS, Callaway CW, Carson AP, et al. Heart disease and stroke statistics-2019 update: a report from the american heart association. Circulation. 2019;139:e56-e528.

4. Liu XS, Zhang ZG, Zhang RL, Gregg S, Morris DC, Wang Y, et al. Stroke induces gene profile changes associated with neurogenesis and angiogenesis in adult subventricular zone progenitor cells. J Cereb Blood Flow Metab. 2007;27:564-74.

5. Krupinski J, Kaluza J, Kumar P, Kumar S, Wang JM. Role of angiogenesis in patients with cerebral ischemic stroke. Stroke. 1994;25:1794-8.

6. Jiang $X$, Suenaga J, Pu H, Wei Z, Smith AD, Hu X, et al. Post-stroke administration of omega-3 polyunsaturated fatty acids promotes neurovascular restoration after ischemic stroke in mice: efficacy declines with aging. Neurobiol Dis. 2019;126:62-75.

7. Manoonkitiwongsa PS, Jackson-Friedman C, McMillan PJ, Schultz RL, Lyden PD. Angiogenesis after stroke is correlated with increased numbers of macrophages: the clean-up hypothesis. J Cereb Blood Flow Metab. 2001;21: 1223-31.

8. Marei HE, Hasan A, Rizzi R, Althani A, Afifi N, Cenciarelli C, et al. Potential of stem cell-based therapy for ischemic stroke. Front Neurol. 2018;9:34.

9. Dao M, Tate CC, McGrogan M, Case CC. Comparing the angiogenic potency of naive marrow stromal cells and notch-transfected marrow stromal cells. J Transl Med. 2013;11:81.

10. Noiseux N, Gnecchi M, Lopez-llasaca M, Zhang L, Solomon SD, Deb A, et al. Mesenchymal stem cells overexpressing akt dramatically repair infarcted myocardium and improve cardiac function despite infrequent cellular fusion or differentiation. Mol Ther. 2006;14:840-50.

11. Lu D, Chen B, Liang Z, Deng W, Jiang Y, Li S, et al. Comparison of bone marrow mesenchymal stem cells with bone marrow-derived mononuclear cells for treatment of diabetic critical limb ischemia and foot ulcer: a double-blind, randomized, controlled trial. Diabetes Res Clin Pract. 2011;92: 26-36.

12. Abdal Dayem A, Lee SB, Kim K, Lim KM, Jeon TI, Seok J, et al. Production of mesenchymal stem cells through stem cell reprogramming. Int J Mol Sci. 2019;20(8):1922.

13. Venkat P, Chen J, Chopp M. Exosome-mediated amplification of endogenous brain repair mechanisms and brain and systemic organ interaction in modulating neurological outcome after stroke. J Cereb Blood Flow Metab. 2018:271678X18782789.

14. Takahashi K, Yamanaka S. Induction of pluripotent stem cells from mouse embryonic and adult fibroblast cultures by defined factors. Cell. 2006;126: 663-76.

15. Jiang B, Yan L, Wang X, Li E, Murphy K, Vaccaro K, et al. Concise review: Mesenchymal stem cells derived from human pluripotent cells, an unlimited and quality-controllable source for therapeutic applications. Stem Cells. 2019;37:572-81.

16. Hu GW, Li Q, Niu X, Hu B, Liu J, Zhou SM, et al. Exosomes secreted by human-induced pluripotent stem cell-derived mesenchymal stem cells attenuate limb ischemia by promoting angiogenesis in mice Stem Cell Res Ther 2015;6:10

17. Giuliani M, Oudrhiri N, Noman ZM, Vernochet A, Chouaib S, Azzarone B, et al. Human mesenchymal stem cells derived from induced pluripotent stem cells down-regulate nk-cell cytolytic machinery. Blood. 2011;118:325462.

18. Hynes K, Menicanin D, Han J, Marino V, Mrozik K, Gronthos S, et al. Mesenchymal stem cells from ips cells facilitate periodontal regeneration. J Dent Res. 2013;92:833-9.

19. Zhou Y, Yamamoto Y, Xiao Z, Ochiya T. The immunomodulatory functions of mesenchymal stromal/stem cells mediated via paracrine activity. J Clin Med. 2019;8(7):1025.

20. Park WS, Ahn SY, Sung SI, Ahn JY, Chang YS. Strategies to enhance paracrine potency of transplanted mesenchymal stem cells in intractable neonatal disorders. Pediatr Res. 2018;83:214-22.
21. Zhang ZG, Buller B, Chopp M. Exosomes - beyond stem cells for restorative therapy in stroke and neurological injury. Nat Rev Neurol. 2019;15:193-203.

22. Kishore R, Khan M. More than tiny sacks: stem cell exosomes as cell-free modality for cardiac repair. Circ Res. 2016;118:330-43.

23. Doeppner TR, Herz J, Gorgens A, Schlechter J, Ludwig AK, Radtke S, et al. Extracellular vesicles improve post-stroke neuroregeneration and prevent postischemic immunosuppression. Stem Cells Transl Med. 2015;4:1131-43.

24. Zhang ZG, Chopp M. Neurorestorative therapies for stroke: underlying mechanisms and translation to the clinic. Lancet Neurol. 2009;8:491-500.

25. Hassanpour M, Rezabakhsh A, Pezeshkian M, Rahbarghazi R, Nouri M. Distinct role of autophagy on angiogenesis: highlights on the effect of autophagy in endothelial lineage and progenitor cells. Stem Cell Res Ther. 2018;9:305.

26. Li H, Wu J, Shen H, Yao X, Liu C, Pianta S, et al. Autophagy in hemorrhagic stroke: mechanisms and clinical implications. Prog Neurobiol. 2018;163-164: 79-97.

27. Shi R, Weng J, Zhao L, Li XM, Gao TM, Kong J. Excessive autophagy contributes to neuron death in cerebral ischemia. CNS Neurosci Ther. 2012; 18:250-60.

28. Li H, Gao A, Feng D, Wang Y, Zhang L, Cui Y, et al. Evaluation of the protective potential of brain microvascular endothelial cell autophagy on blood-brain barrier integrity during experimental cerebral ischemiareperfusion injury. Transl Stroke Res. 2014;5:618-26.

29. Han F, Chen YX, Lu YM, Huang JY, Zhang GS, Tao RR, et al. Regulation of the ischemia-induced autophagy-lysosome processes by nitrosative stress in endothelial cells. J Pineal Res. 2011;51:124-35.

30. Chau YP, Lin SY, Chen JH, Tai MH. Endostatin induces autophagic cell death in eahy926 human endothelial cells. Histol Histopathol. 2003;18:715-26.

31. Liao J, Wu Z, Wang Y, Cheng L, Cui C, Gao Y, et al. Enhanced efficiency of generating induced pluripotent stem (ips) cells from human somatic cells by a combination of six transcription factors. Cell Res. 2008;18:600-3.

32. Chen B, Sun Y, Zhang J, Zhu Q, Yang Y, Niu X, et al. Human embryonic stem cell-derived exosomes promote pressure ulcer healing in aged mice by rejuvenating senescent endothelial cells. Stem Cell Res Ther. 2019;10:142.

33. Tian Y, Ma L, Gong M, Su G, Zhu S, Zhang W, et al. Protein profiling and sizing of extracellular vesicles from colorectal cancer patients via flow cytometry. ACS Nano. 2018;12:671-80.

34. Zhang H, Xia Y, Ye Q, Yu F, Zhu W, Li P, et al. In vivo expansion of regulatory t cells with IL-2//L-2 antibody complex protects against transient ischemic stroke. J Neurosci. 2018;38(47):10168-79.

35. Ling X, Zhang G, Xia Y, Zhu Q, Zhang J, Li Q, et al. Exosomes from human urine-derived stem cells enhanced neurogenesis via mir-26a/hdac6 axis after ischaemic stroke. J Cell Mol Med. 2020;24:640-54.

36. Wiklander OP, Nordin JZ, O'Loughlin A, Gustafsson Y, Corso G, Mager I, et al. Extracellular vesicle in vivo biodistribution is determined by cell source, route of administration and targeting. J Extracell Vesicles. 2015;4:26316.

37. Zhang L, Chopp M, Lu M, Zhang T, Li C, Winter S, et al. Demonstration of therapeutic window of cerebrolysin in embolic stroke: a prospective, randomized, blinded, and placebo-controlled study. Int I Stroke. 2017;12:628-35.

38. Fei X, Zhang X, Wang Q, Li J, Shen H, Wang X, et al. Xijiao dihuang decoction alleviates ischemic brain injury in mcao rats by regulating inflammation, neurogenesis, and angiogenesis. Evid Based Complement Alternat Med. 2018:2018:5945128.

39. You L, Wang Z, Li H, Shou J, Jing Z, Xie J, et al. The role of stat3 in autophagy. Autophagy. 2015;11:729-39.

40. Arai K, Jin G, Navaratna D, Lo EH. Brain angiogenesis in developmental and pathological processes: neurovascular injury and angiogenic recovery after stroke. FEBS J. 2009;276:4644-52.

41. Brumm AJ, Carmichael ST. Not just a rush of blood to the head. Nat Med. 2012;18:1609-10.

42. Cai $M$, Zhang $W$, Weng Z, Stetler RA, Jiang $X$, Shi $Y$, et al. Promoting neurovascular recovery in aged mice after ischemic stroke - prophylactic effect of omega-3 polyunsaturated fatty acids. Aging Dis. 2017;8:531-45.

43. Li L, Chu L, Ren C, Wang J, Sun S, Li T, et al. Enhanced migration of bone marrow-derived mesenchymal stem cells with tetramethylpyrazine and its synergistic effect on angiogenesis and neurogenesis after cerebral ischemia in rats. Stem Cells Dev. 2019;28:871-81.

44. Todeschi MR, El Backly R, Capelli C, Daga A, Patrone E, Introna M, et al. Transplanted umbilical cord mesenchymal stem cells modify the in vivo microenvironment enhancing angiogenesis and leading to bone regeneration. Stem Cells Dev. 2015;24:1570-81. 
45. Brennan MA, Renaud A, Guilloton F, Mebarki M, Trichet V, Sensebe L, et al. Inferior in vivo osteogenesis and superior angiogenesis of human adiposederived stem cells compared with bone marrow-derived stem cells cultured in xeno-free conditions. Stem Cells Transl Med. 2017;6:2160-72.

46. Liu X, Li Q, Niu X, Hu B, Chen S, Song W, et al. Exosomes secreted from human-induced pluripotent stem cell-derived mesenchymal stem cells prevent osteonecrosis of the femoral head by promoting angiogenesis. Int J Biol Sci. 2017;13:232-44.

47. Thiebaut AM, Hedou E, Marciniak SJ, Vivien D, Roussel BD. Proteostasis during cerebral ischemia. Front Neurosci. 2019;13:637.

48. Buckley KM, Hess DL, Sazonova IY, Periyasamy-Thandavan S, Barrett JR, Kirks $R$, et al. Rapamycin up-regulation of autophagy reduces infarct size and improves outcomes in both permanent mcal, and embolic mcao, murine models of stroke. Exp Transl Stroke Med. 2014;6:8.

49. Ryan F, Khodagholi F, Dargahi L, Minai-Tehrani D, Ahmadiani A. Temporal pattern and crosstalk of necroptosis markers with autophagy and apoptosis associated proteins in ischemic hippocampus. Neurotox Res. 2018;34:79-92.

50. Cui DR, Wang L, Jiang W, Qi AH, Zhou QH, Zhang XL. Propofol prevents cerebral ischemia-triggered autophagy activation and cell death in the rat hippocampus through the nf-kappab/p53 signaling pathway. Neuroscience. 2013;246:117-32

51. Wang P, Shao BZ, Deng Z, Chen S, Yue Z, Miao CY. Autophagy in ischemic stroke. Prog Neurobiol. 2018;163-164:98-117.

52. Du JH, Li X, Li R, Cheng BX, Kuerbanjiang M, Ma L. Role of autophagy in angiogenesis induced by a high-glucose condition in rf/6a cells. Ophthalmologica. 2017;237:85-95.

53. Rezabakhsh A, Ahmadi M, Khaksar M, Montaseri A, Malekinejad H, Rahbarghazi $R$, et al. Rapamycin inhibits oxidative/nitrosative stress and enhances angiogenesis in high glucose-treated human umbilical vein endothelial cells: role of autophagy. Biomed Pharmacother. 2017;93:885-94.

54. Li X, Lu Q, Xie W, Wang Y, Wang G. Anti-tumor effects of triptolide on angiogenesis and cell apoptosis in osteosarcoma cells by inducing autophagy via repressing wnt/beta-catenin signaling. Biochem Biophys Res Commun. 2018:496:443-9.

55. Kumar S, Guru SK, Pathania AS, Kumar A, Bhushan S, Malik F. Autophagy triggered by magnolol derivative negatively regulates angiogenesis. Cell Death Dis. 2013;4:e889.

56. Liu H, Yu S, Zhang $H, X u J$. Angiogenesis impairment in diabetes: role of methylglyoxal-induced receptor for advanced glycation endproducts, autophagy and vascular endothelial growth factor receptor 2 . PLoS One 2012;7:e46720

57. Ebrahim N, Ahmed IA, Hussien NI, et al. Mesenchymal stem cell-derived exosomes ameliorated diabetic nephropathy by autophagy induction through the mTOR signaling pathway. Cells. 2018;7(12):226.

58. Yang Y, Li Y, Chen X, Cheng X, Liao Y, Yu X. Exosomal transfer of mir-30a between cardiomyocytes regulates autophagy after hypoxia. J Mol Med (Berl). 2016;94:711-24.

59. Zhang L, Song Y, Chen L, Li D, Feng H, Lu Z, et al. Mir-20a-containing exosomes from umbilical cord mesenchymal stem cells alleviates liver ischemia/reperfusion injury. J Cell Physiol. 2020;235:3698-710.

60. Liu J, Jiang M, Deng S, Lu J, Huang H, Zhang Y, et al. Mir-93-5p-containing exosomes treatment attenuates acute myocardial infarction-induced myocardial damage. Mol Ther Nucleic Acids. 2018;11:103-15.

61. Xiao C, Wang K, Xu Y, Hu H, Zhang N, Wang Y, et al. Transplanted mesenchymal stem cells reduce autophagic flux in infarcted hearts via the exosomal transfer of mir-125b. Circ Res. 2018;123:564-78.

62. Gong XH, Liu H, Wang SJ, Liang SW, Wang GG. Exosomes derived from sdf1-overexpressing mesenchymal stem cells inhibit ischemic myocardial cell apoptosis and promote cardiac endothelial microvascular regeneration in mice with myocardial infarction. J Cell Physiol. 2019;234:13878-93.

63. Hoffmann CJ, Harms U, Rex A, Szulzewsky F, Wolf SA, Grittner U, et al. Vascular signal transducer and activator of transcription-3 promotes angiogenesis and neuroplasticity long-term after stroke. Circulation. 2015; 131:1772-82.

64. Nakagawa S, Aruga J. Sphingosine 1-phosphate signaling is involved in impaired blood-brain barrier function in ischemia-reperfusion injury. Mol Neurobiol. 2020;57:1594-606.

65. Liang Z, Wu G, Fan C, Xu J, Jiang S, Yan X, et al. The emerging role of signa transducer and activator of transcription 3 in cerebral ischemic and hemorrhagic stroke. Prog Neurobiol. 2016;137:1-16.

\section{Publisher's Note}

Springer Nature remains neutral with regard to jurisdictional claims in published maps and institutional affiliations.

\section{Ready to submit your research? Choose BMC and benefit from:}

- fast, convenient online submission

- thorough peer review by experienced researchers in your field

- rapid publication on acceptance

- support for research data, including large and complex data types

- gold Open Access which fosters wider collaboration and increased citations

- maximum visibility for your research: over $100 \mathrm{M}$ website views per year

At BMC, research is always in progress.

Learn more biomedcentral.com/submissions 